\title{
Leonard triples and hypercubes
}

\author{
Štefko Miklavič
}

Received: 3 May 2007 / Accepted: 14 November 2007 / Published online: 30 November 2007

(C) Springer Science+Business Media, LLC 2007

\begin{abstract}
Let $V$ denote a vector space over $\mathbb{C}$ with finite positive dimension. By a Leonard triple on $V$ we mean an ordered triple of linear operators on $V$ such that for each of these operators there exists a basis of $V$ with respect to which the matrix representing that operator is diagonal and the matrices representing the other two operators are irreducible tridiagonal.

Let $D$ denote a positive integer and let $Q_{D}$ denote the graph of the $D$-dimensional hypercube. Let $X$ denote the vertex set of $Q_{D}$ and let $A \in \operatorname{Mat}_{X}(\mathbb{C})$ denote the adjacency matrix of $Q_{D}$. Fix $x \in X$ and let $A^{*} \in \operatorname{Mat}_{X}(\mathbb{C})$ denote the corresponding dual adjacency matrix. Let $T$ denote the subalgebra of $\operatorname{Mat}_{X}(\mathbb{C})$ generated by $A, A^{*}$. We refer to $T$ as the Terwilliger algebra of $Q_{D}$ with respect to $x$. The matrices $A$ and $A^{*}$ are related by the fact that $2 \mathbf{i} A=A^{*} A^{\varepsilon}-A^{\varepsilon} A^{*}$ and $2 \mathbf{i} A^{*}=A^{\varepsilon} A-A A^{\varepsilon}$, where $2 \mathbf{i} A^{\varepsilon}=A A^{*}-A^{*} A$ and $\mathbf{i}^{2}=-1$.

We show that the triple $A, A^{*}, A^{\varepsilon}$ acts on each irreducible $T$-module as a Leonard triple. We give a detailed description of these Leonard triples.
\end{abstract}

Keywords Leonard triple · Distance-regular graph · Hypercube $\cdot$ Terwilliger algebra

The author gratefully acknowledges support by the US Department of State and the Fulbright Scholar Program, and thanks the University of Wisconsin-Madison for hospitality during his visit in Spring 2007. Supported in part by "Javna agencija za raziskovalno dejavnost Republike Slovenije", program no. Z1-9614.

S̆. Miklavič $(\bowtie)$

Department of Mathematics and Computer Science, Faculty of Education, University of Primorska, 6000 Koper, Slovenia

e-mail: miklavic@pef.upr.si 


\section{Introduction}

We start by recalling the definition of a Leonard pair. To describe this object we use the following terms. Let $\mathbb{C}$ denote the field of complex numbers. A square matrix with entries in $\mathbb{C}$ is called tridiagonal whenever each nonzero entry lies on either the diagonal, the subdiagonal, or the superdiagonal. A tridiagonal matrix is called irreducible whenever each entry on the subdiagonal is nonzero and each entry on the superdiagonal is nonzero.

Definition 1.1 [21, Definition 1.1] Let $V$ denote a vector space over $\mathbb{C}$ with finite positive dimension. By a Leonard pair on $V$ we mean an ordered pair of linear operators $A: V \rightarrow V$ and $A^{*}: V \rightarrow V$ which satisfy the conditions (i), (ii) below.

(i) There exists a basis for $V$ with respect to which the matrix representing $A$ is diagonal and the matrix representing $A^{*}$ is irreducible tridiagonal.

(ii) There exists a basis for $V$ with respect to which the matrix representing $A^{*}$ is diagonal and the matrix representing $A$ is irreducible tridiagonal.

Leonard pairs have been explored as linear algebraic objects, in connection with orthogonal polynomials, and as representations of certain algebras [17-27]. The notion of a Leonard triple was introduced by Curtin in [5]. We recall the definition.

Definition 1.2 [5, Definition 1.2] Let $V$ denote a vector space over $\mathbb{C}$ with finite positive dimension. By a Leonard triple on $V$ we mean an ordered triple of linear operators $A: V \rightarrow V, A^{*}: V \rightarrow V, A^{\varepsilon}: V \rightarrow V$ which satisfy the conditions (i)(iii) below.

(i) There exists a basis for $V$ with respect to which the matrix representing $A$ is diagonal and the matrices representing $A^{*}$ and $A^{\varepsilon}$ are each irreducible tridiagonal.

(ii) There exists a basis for $V$ with respect to which the matrix representing $A^{*}$ is diagonal and the matrices representing $A^{\varepsilon}$ and $A$ are each irreducible tridiagonal.

(iii) There exists a basis for $V$ with respect to which the matrix representing $A^{\varepsilon}$ is diagonal and the matrices representing $A$ and $A^{*}$ are each irreducible tridiagonal.

Leonard triples are closely related to Leonard pairs. Indeed, any ordered pair of distinct elements of a Leonard triple form a Leonard pair. This allows us to take advantage of the literature concerning Leonard pairs in our study of Leonard triples. The isomorphism classes of Leonard pairs are in bijective correspondence with the polynomials in the terminating branch of Askey-Wilson scheme [24, 25]. In particular, results concerning Leonard pairs also have interpretations as results concerning such polynomials. Consequently, results concerning Leonard triples also have interpretations as results concerning such polynomials. Leonard pairs play a role in representation theory $[12,14,20,21,28]$ and combinatorics $[4,7,8,10,12,17-19$, 23]. Consequently, also Leonard triples play a role in representation theory and combinatorics. Leonard triples are also related to spin models [6], generalized Markov problem in number theory and the Poncelet problem in projective geometry [15].

In this paper we consider a situation in graph theory where Leonard triples arise naturally. The situation is described as follows. Let $D$ denote a positive integer, let 
$Q_{D}$ denote the graph of the hypercube with dimension $D$ (see Section 4 for formal definitions), and let $X$ denote the vertex set of $Q_{D}$. Let $\operatorname{Mat}_{X}(\mathbb{C})$ denote the $\mathbb{C}$ algebra of matrices with entries in $\mathbb{C}$ and with rows and columns indexed by $X$. Let $A \in \operatorname{Mat}_{X}(\mathbb{C})$ denote the adjacency matrix of $Q_{D}$. For the rest of this introduction fix $x \in X$. Let $A^{*}=A^{*}(x)$ denote the diagonal matrix in $\operatorname{Mat}_{X}(\mathbb{C})$ with $(y, y)$-entry $D-2 \partial(x, y)$ for $y \in X$, where $\partial$ denotes path-length distance. The matrix $A^{*}$ is called the dual adjacency matrix of $Q_{D}$ with respect to $x$ [17]. Let $T=T(x)$ denote the subalgebra of $\operatorname{Mat}_{X}(\mathbb{C})$ generated by $A, A^{*}$. The algebra $T$ is known as the Terwilliger algebra of $Q_{D}$ with respect to $x$ [17]. As we shall see, $A$ and $A^{*}$ are related by the fact that $2 \mathbf{i} A=A^{*} A^{\varepsilon}-A^{\varepsilon} A^{*}$ and $2 \mathbf{i} A^{*}=A^{\varepsilon} A-A A^{\varepsilon}$, where $2 \mathbf{i} A^{\varepsilon}=A A^{*}-A^{*} A$ and $\mathbf{i}^{2}=-1$. We call $A^{\varepsilon}$ the imaginary adjacency matrix of $Q_{D}$ with respect to $x$. The matrices $A, A^{*}, A^{\varepsilon}$ are similar; indeed we display an invertible matrix $P \in T$ such that $A^{*}=P A P^{-1}, A^{\varepsilon}=P A^{*} P^{-1}, A=P A^{\varepsilon} P^{-1}$.

Let $W$ denote an irreducible $T$-module. We show that the triple $A, A^{*}, A^{\varepsilon}$ acts on $W$ as a Leonard triple. We give this triple a detailed description which is summarized as follows. Consider the three bases for $W$ afforded by Definition 1.2. For each of these bases we display two normalizations that we find attractive, and this yields six bases for $W$. We compute the matrices which represent $A, A^{*}, A^{\varepsilon}$ with respect to these six bases. We display the inner products between each pair of these bases. We then display the transition matrices between each pair of these bases. We remark that our paper extends the work of Go [10].

\section{Preliminaries}

In this section we review some definitions and basic results concerning distanceregular graphs. See the book of Brouwer, Cohen and Neumaier [3] for more background information.

Let $X$ denote a nonempty finite set. Let $\operatorname{Mat}_{X}(\mathbb{C})$ denote the $\mathbb{C}$-algebra of matrices with entries in $\mathbb{C}$ and with rows and columns indexed by $X$. For $B \in \operatorname{Mat}_{X}(\mathbb{C})$ let $B^{t}$ and $\bar{B}$ denote the transpose and the complex conjugate of $B$, respectively. Let $V=$ $\mathbb{C}^{X}$ denote the vector space over $\mathbb{C}$ consisting of column vectors with entries in $\mathbb{C}$ and rows indexed by $X$. We observe $\operatorname{Mat}_{X}(\mathbb{C})$ acts on $V$ by left multiplication. We refer to $V$ as the standard module of $\operatorname{Mat}_{X}(\mathbb{C})$. For $v \in V$ let $v^{t}$ and $\bar{v}$ denote the transpose and the complex conjugate of $v$, respectively. We endow $V$ with the Hermitean inner product $\langle u, v\rangle=u^{t} \bar{v}(u, v \in V)$. For $y \in X$ let $\hat{y}$ denote the vector in $V$ with a 1 in the $y$ coordinate and 0 in all other coordinates. Observe that $\{\hat{y} \mid y \in X\}$ is an orthogonal basis for $V$. The following will be useful: for each $B \in \operatorname{Mat}_{X}(\mathbb{C})$ we have

$$
\langle u, B v\rangle=\left\langle\bar{B}^{t} u, v\right\rangle \quad(u, v \in V) .
$$

Let $\Gamma=(X, R)$ denote a finite, undirected, connected graph, without loops or multiple edges, with vertex set $X$, edge set $R$, path-length distance function $\partial$, and diameter $D:=\max \{\partial(x, y) \mid x, y \in X\}$. For a vertex $x \in X$ and an integer $i \geq 0$ let $\Gamma_{i}(x)$ denote the set of vertices at distance $i$ from $x$. For an integer $k \geq 0$ we say $\Gamma$ is regular with valency $k$ whenever $\left|\Gamma_{1}(x)\right|=k$ for all $x \in X$. We say $\bar{\Gamma}$ is distanceregular whenever for all integers $0 \leq h, i, j \leq D$ and all $x, y \in X$ with $\partial(x, y)=h$ 
the number

$$
p_{i j}^{h}:=\left|\Gamma_{i}(x) \cap \Gamma_{j}(y)\right|
$$

is independent of $x, y$. The constants $p_{i j}^{h}$ are known as the intersection numbers of $\Gamma$. From now on we assume $\Gamma$ is distance-regular with $D \geq 1$. For convenience set $c_{i}:=p_{1, i-1}^{i}(1 \leq i \leq D), a_{i}:=p_{1 i}^{i}(0 \leq i \leq D), b_{i}:=p_{1, i+1}^{i}(0 \leq i \leq D-1), k_{i}:=$ $p_{i i}^{0}(0 \leq i \leq D)$, and $c_{0}=0, b_{D}=0$. We observe that $\Gamma$ is regular with valency $k=k_{1}=b_{0}$ and that $c_{i}+a_{i}+b_{i}=k$ for $0 \leq i \leq D$. By [3, p. 127] the following hold for $0 \leq h, i, j \leq D$ : (i) $p_{i j}^{h}=0$ if one of $h, i, j$ is greater than the sum of the other two; and (ii) $p_{i j}^{h} \neq 0$ if one of $h, i, j$ equals the sum of the other two.

We now recall the Bose-Mesner algebra of $\Gamma$. For $0 \leq i \leq D$ let $A_{i}$ denote the matrix in $\operatorname{Mat}_{X}(\mathbb{C})$ with entries

$$
\left(A_{i}\right)_{x y}=\left\{\begin{array}{l}
1 \text { if } \partial(x, y)=i, \\
0 \text { if } \partial(x, y) \neq i
\end{array} \quad(x, y \in X) .\right.
$$

We abbreviate $A=A_{1}$ and call this the adjacency matrix of $\Gamma$. Let $M$ denote the subalgebra of $\operatorname{Mat}_{X}(\mathbb{C})$ generated by $A$. By $\left[3\right.$, p. 44] the matrices $A_{0}, A_{1}, \ldots, A_{D}$ form a basis for $M$. We call $M$ the Bose-Mesner algebra of $\Gamma$. We observe that $M$ is commutative and semi-simple. By [3, p. 45] there exists a basis $E_{0}, E_{1}, \ldots, E_{D}$ for $M$ such that

$$
\begin{array}{r}
E_{0}=|X|^{-1} J, \\
E_{0}+E_{1}+\cdots+E_{D}=I, \\
E_{i}^{t}=E_{i} \quad(0 \leq i \leq D), \\
\overline{E_{i}}=E_{i}(0 \leq i \leq D), \\
E_{i} E_{j}=\delta_{i j} E_{i}(0 \leq i, j \leq D),
\end{array}
$$

where $I$ and $J$ denote the identity and the all-ones matrix of $\operatorname{Mat}_{X}(\mathbb{C})$, respectively. For convenience we define $E_{i}=0$ if $i<0$ or $i>D$. The matrices $E_{0}, E_{1}, \ldots, E_{D}$ are known as the primitive idempotents of $\Gamma$, and $E_{0}$ is called the trivial idempotent. We recall the eigenvalues of $\Gamma$. Since $E_{0}, E_{1}, \ldots, E_{D}$ is a basis for $M$, there exist scalars $\theta_{0}, \theta_{1}, \ldots, \theta_{D} \in \mathbb{C}$ such that

$$
A=\sum_{i=0}^{D} \theta_{i} E_{i}
$$

Combining this with (2) and (6) we find $A E_{i}=E_{i} A=\theta_{i} E_{i}$ for $0 \leq i \leq D$ and $\theta_{0}=k$. The scalars $\theta_{0}, \theta_{1}, \ldots, \theta_{D}$ are real [2, p. 197]. Observe that $\theta_{0}, \theta_{1}, \ldots, \theta_{D}$ are mutually distinct since $A$ generates $M$. We refer to $\theta_{i}$ as the eigenvalue of $\Gamma$ associated with $E_{i}$. For $0 \leq i \leq D$ let $m_{i}$ denote the rank of $E_{i}$. We call $m_{i}$ the multiplicity of $\theta_{i}$.

By (3)-(6),

$$
V=E_{0} V+E_{1} V+\cdots+E_{D} V \quad \text { (orthogonal direct sum). }
$$


By linear interpolation,

$$
E_{i}=\prod_{\substack{0 \leq j \leq D \\ j \neq i}} \frac{A-\theta_{j} I}{\theta_{i}-\theta_{j}} \quad(0 \leq i \leq D) .
$$

We now recall the $Q$-polynomial property. Note that $A_{i} \circ A_{j}=\delta_{i j} A_{i}$ for $0 \leq i, j \leq$ $D$, where $\circ$ is the entry-wise multiplication. Therefore $M$ is closed under $\circ$. Thus there exist $q_{i j}^{h} \in \mathbb{C}(0 \leq h, i, j \leq D)$ such that

$$
E_{i} \circ E_{j}=|X|^{-1} \sum_{h=0}^{D} q_{i j}^{h} E_{h} \quad(0 \leq i, j \leq D) .
$$

By [3, Proposition 4.1.5] the scalars $q_{i j}^{h}$ are real and nonnegative for $0 \leq h, i, j \leq D$. The $q_{i j}^{h}$ are called the Krein parameters of $\Gamma$. The graph $\Gamma$ is said to be $Q$-polynomial (with respect to the given ordering $E_{0}, E_{1}, \ldots, E_{D}$ of the primitive idempotents) whenever the following hold for $0 \leq h, i, j \leq D$ : (i) $q_{i j}^{h}=0$ if one of $h, i, j$ is greater than the sum of the other two; and (ii) $q_{i j}^{h} \neq 0$ if one of $h, i, j$ equals the sum of the other two.

\section{The Terwilliger algebra}

In this section we recall the dual Bose-Mesner algebra and the Terwilliger algebra of $\Gamma$. For the rest of this section fix $x \in X$. For $0 \leq i \leq D$ let $E_{i}^{*}=E_{i}^{*}(x)$ denote the diagonal matrix in $\operatorname{Mat}_{X}(\mathbb{C})$ with entries

$$
\left(E_{i}^{*}\right)_{y y}=\left\{\begin{array}{l}
1 \text { if } \partial(x, y)=i, \\
0 \text { if } \partial(x, y) \neq i
\end{array} \quad(y \in X) .\right.
$$

We call $E_{i}^{*}$ the $i$ th dual idempotent of $\Gamma$ with respect to $x$. We observe

$$
\begin{array}{r}
E_{0}^{*}+E_{1}^{*}+\cdots+E_{D}^{*}=I, \\
E_{i}^{* t}=E_{i}^{*}(0 \leq i \leq D), \\
\overline{E_{i}^{*}}=E_{i}^{*}(0 \leq i \leq D), \\
E_{i}^{*} E_{j}^{*}=\delta_{i j} E_{i}^{*}(0 \leq i, j \leq D) .
\end{array}
$$

By construction $E_{0}^{*}, E_{1}^{*}, \ldots, E_{D}^{*}$ are linearly independent. Let $M^{*}=M^{*}(x)$ denote the subalgebra of $\operatorname{Mat}_{X}(\mathbb{C})$ spanned by $E_{0}^{*}, E_{1}^{*}, \ldots, E_{D}^{*}$. We call $M^{*}$ the dual BoseMesner algebra of $\Gamma$ with respect to $x$. We observe $M^{*}$ is commutative and semisimple.

Assume $\Gamma$ is $Q$-polynomial with respect to the ordering $E_{0}, E_{1}, \ldots, E_{D}$ of the primitive idempotents. Let $A^{*}=A^{*}(x)$ denote the diagonal matrix in $\operatorname{Mat}_{X}(\mathbb{C})$ with $(y, y)$-entry

$$
A_{y y}^{*}=|X| E_{x y} \quad(y \in X)
$$


where $E=E_{1}$. We call $A^{*}$ the dual adjacency matrix of $\Gamma$ with respect to $x$. By [17, Lemma 3.11] $M^{*}$ is generated by $A^{*}$. We recall the dual eigenvalues of $\Gamma$. Since $E_{0}^{*}, E_{1}^{*}, \ldots, E_{D}^{*}$ is a basis for $M^{*}$ there exist $\theta_{0}^{*}, \theta_{1}^{*}, \ldots, \theta_{D}^{*} \in \mathbb{C}$ such that

$$
A^{*}=\sum_{i=0}^{D} \theta_{i}^{*} E_{i}^{*} .
$$

Combining this with (13) we find $A^{*} E_{i}^{*}=E_{i}^{*} A^{*}=\theta_{i}^{*} E_{i}^{*}(0 \leq i \leq D)$. By [17, Lemma 3.11] $\theta_{0}^{*}, \theta_{1}^{*}, \ldots, \theta_{D}^{*}$ are real. The scalars $\theta_{0}^{*}, \theta_{1}^{*}, \ldots, \theta_{D}^{*}$ are mutually distinct since $A^{*}$ generates $M^{*}$. Note that $\theta_{i}^{*}$ is an eigenvalue of $A^{*}$ and $E_{i}^{*} V$ is the corresponding eigenspace $(0 \leq i \leq D)$. Using (10)-(13) we find

$$
V=E_{0}^{*} V+E_{1}^{*} V+\cdots+E_{D}^{*} V \quad \text { (orthogonal direct sum). }
$$

We call the sequence $\theta_{0}^{*}, \theta_{1}^{*}, \ldots, \theta_{D}^{*}$ the dual eigenvalue sequence of $\Gamma$. Observe that for $0 \leq i \leq D$ the rank of $E_{i}^{*}$ is $k_{i}$. Therefore $k_{i}$ is the multiplicity with which $\theta_{i}^{*}$ appears as an eigenvalue of $A^{*}$.

By linear interpolation we obtain

$$
E_{i}^{*}=\prod_{\substack{0 \leq j \leq D \\ j \neq i}} \frac{A^{*}-\theta_{j}^{*} I}{\theta_{i}^{*}-\theta_{j}^{*}} .
$$

By [17, Lemma 3.2] the following hold for $0 \leq h, j \leq D$ :

$$
\begin{aligned}
& E_{j}^{*} A E_{h}^{*}=0 \text { if and only if } p_{1 j}^{h}=0 ; \\
& E_{j} A^{*} E_{h}=0 \text { if and only if } q_{1 j}^{h}=0 .
\end{aligned}
$$

Let $T=T(x)$ denote the subalgebra of $\operatorname{Mat}_{X}(\mathbb{C})$ generated by $M$ and $M^{*}$. We call $T$ the Terwilliger algebra of $\Gamma$ with respect to $x$ [17, Definition 3.3].

By a $T$-module we mean a subspace $W$ of $V$ such that $B W \subseteq W$ for all $B \in T$. Let $W$ denote a $T$-module. Then $W$ is said to be irreducible whenever $W$ is nonzero and $W$ contains no $T$-modules other than 0 and $W$.

By construction $T$ is closed under the conjugate-transpose map so $T$ is semisimple [17, Lemma 3.4(i)]. By [17, Lemma 3.4(ii)] $V$ decomposes into an orthogonal direct sum of irreducible $T$-modules. Let $W$ denote an irreducible $T$-module. By [17, Lemma 3.4(iii)] $W$ is the orthogonal direct sum of the nonvanishing $E_{i} W(0 \leq i \leq$ $D)$ and the orthogonal direct sum of the nonvanishing $E_{i}^{*} W(0 \leq i \leq D)$. By the endpoint of $W$ we mean $\min \left\{i \mid 0 \leq i \leq D, E_{i}^{*} W \neq 0\right\}$. By the diameter of $W$ we mean $\left|\left\{i \mid 0 \leq i \leq D, E_{i}^{*} W \neq 0\right\}\right|-1$. By the dual endpoint of $W$ we mean $\min \{i \mid 0 \leq$ $\left.i \leq D, E_{i} W \neq 0\right\}$. By the dual diameter of $W$ we mean $\mid\left\{i \mid 0 \leq i \leq D, E_{i} W \neq\right.$ $0\} \mid-1$. By [12, Lemma 4.5] the diameter and the dual diameter of $W$ coincide. Let $r$ and $r^{*}$ denote the endpoint and the dual endpoint of $W$, respectively, and let $d$ denote the diameter of $W$. By [17, Lemma 3.9(ii), Lemma 3.12(ii)] the following hold for $0 \leq i \leq D$ :

$$
E_{i} W \neq 0 \text { if and only if } r^{*} \leq i \leq r^{*}+d,
$$




$$
E_{i}^{*} W \neq 0 \text { if and only if } r \leq i \leq r+d .
$$

Let $W$ denote an irreducible $T$-module. By [17, Lemma 3.9, Lemma 3.12] the following are equivalent: (i) $\operatorname{dim}\left(E_{i} W\right) \leq 1$ for $0 \leq i \leq D$; (ii) $\operatorname{dim}\left(E_{i}^{*} W\right) \leq 1$ for $0 \leq i \leq D$. In this case $W$ is called thin.

\section{The hypercubes}

In this section we recall the hypercube graph and some of its basic properties. Let $D$ denote a positive integer, and let $\{0,1\}^{D}$ denote the set of sequences $\left(t_{1}, t_{2}, \ldots, t_{D}\right)$, where $t_{i} \in\{0,1\}$ for $1 \leq i \leq D$. Let $Q_{D}$ denote the graph with vertex set $X=\{0,1\}^{D}$, and where two vertices are adjacent if and only if they differ in exactly one coordinate. We call $Q_{D}$ the $D$-cube or a hypercube. The graph $Q_{D}$ is connected and for $y, z \in X$ the distance $\partial(y, z)$ is the number of coordinates at which $y$ and $z$ differ. In particular the diameter of $Q_{D}$ equals $D$. The graph $Q_{D}$ is bipartite with bipartition $X=X^{+} \cup X^{-}$, where $X^{+}$(resp. $X^{-}$) is the set of vertices of $Q_{D}$ with an even (resp. odd) number of positive coordinates. By [3, p. 261] $Q_{D}$ is distance-regular with intersection numbers

$$
a_{i}=0, \quad b_{i}=D-i, \quad c_{i}=i, \quad k_{i}=\left(\begin{array}{c}
D \\
i
\end{array}\right) \quad(0 \leq i \leq D) .
$$

Let $\theta_{0}>\cdots>\theta_{D}$ denote the eigenvalues of $Q_{D}$. By [3, p. 261] these eigenvalues and their multiplicities are given by

$$
\theta_{i}=D-2 i, \quad m_{i}=\left(\begin{array}{c}
D \\
i
\end{array}\right) \quad(0 \leq i \leq D) .
$$

For $0 \leq i \leq D$ let $E_{i}$ denote the primitive idempotent of $Q_{D}$ associated with $\theta_{i}$. By [3, Corollary 8.4.2], $Q_{D}$ is $Q$-polynomial with respect to $E_{0}, E_{1}, \ldots, E_{D}$. Moreover, it follows from [3, Theorem 8.4.4] that

$$
p_{i j}^{h}=q_{i j}^{h} \quad(0 \leq h, i, j \leq D) .
$$

Since $Q_{D}$ is bipartite,

$$
p_{i j}^{h}=0 \text { if } h+i+j \text { is odd }(0 \leq h, i, j \leq D) .
$$

Let $\theta_{0}^{*}, \ldots, \theta_{D}^{*}$ denote the dual eigenvalue sequence of $Q_{D}$ for the given $Q$ polynomial structure. Then $\theta_{i}^{*}=\theta_{i}(0 \leq i \leq D)$ [10, Lemma 3.7]. Fix $x \in X$. Let $A^{*}=A^{*}(x)$ denote the corresponding dual adjacency matrix, and let $T=T(x)$ denote the corresponding Terwilliger algebra. By (15) and since $\theta_{i}^{*}=D-2 i$ we have

$$
A_{y y}^{*}=D-2 \partial(x, y) \quad(y \in X) .
$$

Since $Q_{D}$ is vertex-transitive the isomorphism class of $T(x)$ does not dependent on $x$. For notational convenience, for the rest of this paper we assume $x=(0,0, \ldots, 0)$. By [10, Theorem 4.2] we have

$$
A^{* 2} A-2 A^{*} A A^{*}+A A^{* 2}=4 A,
$$




$$
A^{2} A^{*}-2 A A^{*} A+A^{*} A^{2}=4 A^{*} .
$$

Let $W$ denote an irreducible $T$-module. By [10, Theorem 6.3] $W$ is thin. By [10, Theorem 6.3, Theorem 8.1] the endpoint and the dual endpoint of $W$ coincide. Denoting this common value by $r$ we have $d=D-2 r$ and $0 \leq r \leq D / 2$, where $d$ is the diameter of $W[10$, Theorem 6.3].

\section{The Cartesian product and the Kronecker product}

In this section we recall the Cartesian product of graphs and the Kronecker product of matrices. For graphs $\Gamma=(X, R)$ and $\Gamma^{\prime}=\left(X^{\prime}, R^{\prime}\right)$ let $\Gamma \times \Gamma^{\prime}$ denote the graph with vertex set $X \times X^{\prime}$, and with vertex $\left(u, u^{\prime}\right)$ being adjacent to vertex $\left(v, v^{\prime}\right)$ if and only if either $u=v$ and $u^{\prime}$ is adjacent to $v^{\prime}$ in $\Gamma^{\prime}$, or $u^{\prime}=v^{\prime}$ and $u$ is adjacent to $v$ in $\Gamma$. We call $\Gamma \times \Gamma^{\prime}$ the Cartesian product of $\Gamma$ and $\Gamma^{\prime}$.

For $B \in \operatorname{Mat}_{X}(\mathbb{C})$ and $B^{\prime} \in \operatorname{Mat}_{X^{\prime}}(\mathbb{C})$ let $B \otimes B^{\prime}$ denote the matrix in Mat $\operatorname{Mx}_{X X^{\prime}}(\mathbb{C})$, with a $\left(\left(u, u^{\prime}\right),\left(v, v^{\prime}\right)\right)$-entry equal to the $(u, v)$-entry of $B$ times the $\left(u^{\prime}, v^{\prime}\right)$-entry of $B^{\prime}$. We call $B \otimes B^{\prime}$ the Kronecker product of $B$ and $B^{\prime}$. By [9, p. 107]

$$
\left(B_{1} \otimes B_{1}^{\prime}\right)\left(B_{2} \otimes B_{2}^{\prime}\right)=\left(B_{1} B_{2}\right) \otimes\left(B_{1}^{\prime} B_{2}^{\prime}\right) .
$$

Also by [9, p. 107]

$$
\begin{aligned}
& B \otimes\left(\gamma_{1} B_{1}^{\prime}+\gamma_{2} B_{2}^{\prime}\right)=\gamma_{1} B \otimes B_{1}^{\prime}+\gamma_{2} B \otimes B_{2}^{\prime}, \\
& \left(\gamma_{1} B_{1}^{\prime}+\gamma_{2} B_{2}^{\prime}\right) \otimes B=\gamma_{1} B_{1}^{\prime} \otimes B+\gamma_{2} B_{2}^{\prime} \otimes B,
\end{aligned}
$$

where $\gamma_{1}, \gamma_{2} \in \mathbb{C}$. It is also known that

$$
\left(B \otimes B^{\prime}\right)^{t}=B^{t} \otimes B^{\prime t}, \quad \overline{B \otimes B^{\prime}}=\bar{B} \otimes \overline{B^{\prime}} .
$$

For a matrix $B \in \operatorname{Mat}_{X}(\mathbb{C})$ and an integer $r \geq 0$ let $B^{\otimes r}$ denote $B \otimes B \otimes \cdots \otimes B(r$ copies). We interpret $B^{\otimes 0}=1$. Let $\Gamma$ and $\Gamma^{\prime}$ be graphs with adjacency matrices $A$ and $A^{\prime}$, respectively. By construction the adjacency matrix of $\Gamma \times \Gamma^{\prime}$ is equal to $A \otimes$ $I^{\prime}+I \otimes A^{\prime}$, where $I$ and $I^{\prime}$ are the identity matrices of appropriate dimensions (see, for example, [11, Section 12.4]). The hypercube $Q_{D}$ can be viewed as a Cartesian product $Q_{1} \times Q_{1} \times \cdots \times Q_{1}$ ( $D$ copies). By a simple induction argument we find that the adjacency matrix $A$ of $Q_{D}$ satisfies

$$
A=\sum_{i=0}^{D-1} I_{1}^{\otimes i} \otimes A_{1} \otimes I_{1}^{\otimes(D-1-i)},
$$

where $A_{1}$ and $I_{1}$ denote the adjacency matrix of $Q_{1}$ and the $2 \times 2$ identity matrix, respectively. The reader is invited to verify that a similar equation holds for the dual adjacency matrix $A^{*}$ of the hypercube $Q_{D}$ :

$$
A^{*}=\sum_{i=0}^{D-1} I_{1}^{\otimes i} \otimes A_{1}^{*} \otimes I_{1}^{\otimes(D-1-i)} .
$$




\section{The imaginary adjacency matrix of $Q_{D}$}

In this section we define the imaginary adjacency matrix of the hypercube $Q_{D}$. We use the following notation.

Notation 6.1 Let $D$ denote a positive integer and let $Q_{D}=(X, R)$ denote the $D$ cube. Let $A$ denote the adjacency matrix of $Q_{D}$ and let $I$ denote the identity matrix in $\operatorname{Mat}_{X}(\mathbb{C})$. Fix $x=(0,0, \ldots, 0) \in X$, and let $A^{*}=A^{*}(x)$ and $T=T(x)$ denote the corresponding dual adjacency matrix and the Terwilliger algebra, respectively. We define the matrix $A^{\varepsilon}=A^{\varepsilon}(x)$ by

$$
A^{\varepsilon}=-\mathbf{i}\left(A A^{*}-A^{*} A\right) / 2,
$$

where $\mathbf{i}^{2}=-1$.

Note that $A^{\varepsilon} \in T$. We have the following observation.

Lemma 6.2 With reference to Notation 6.1, for $y, z \in X$ the $(y, z)$-entry of $A^{\varepsilon}$ is given by

$$
A_{y z}^{\varepsilon}=\mathbf{i}(\partial(x, z)-\partial(x, y)) A_{y z} .
$$

Proof. Since $A^{*}$ is diagonal we obtain $\left(A A^{*}\right)_{y z}=A_{y z} A_{z z}^{*}$ and $\left(A^{*} A\right)_{y z}=A_{y y}^{*} A_{y z}$. By (26) $A_{y y}^{*}=D-2 \partial(x, y)$ and $A_{z z}^{*}=D-2 \partial(x, z)$. The result now follows using (35).

Motivated by Lemma 6.2 we call $A^{\varepsilon}$ the imaginary adjacency matrix of $Q_{D}$ with respect to $x$. The following result will be useful.

Lemma 6.3 With reference to Notation 6.1 the following (i)-(iii) hold.

(i) $A A^{*}-A^{*} A=2 \mathbf{i} A^{\varepsilon}$,

(ii) $A^{*} A^{\varepsilon}-A^{\varepsilon} A^{*}=2 \mathbf{i} A$,

(iii) $A^{\varepsilon} A-A A^{\varepsilon}=2 \mathbf{i} A^{*}$.

PROOF. (i) Immediate from (35).

(ii), (iii) Eliminate $A^{\varepsilon}$ using (35) and simplify using (27) and (28).

Lemma 6.4 With reference to Notation 6.1 we have

$$
A^{\varepsilon}=\sum_{i=0}^{D-1} I_{1}^{\otimes i} \otimes A_{1}^{\varepsilon} \otimes I_{1}^{\otimes(D-1-i)},
$$

where $A_{1}^{\varepsilon}$ denotes the imaginary adjacency matrix of $Q_{1}$ and $I_{1}$ denotes the $2 \times 2$ identity matrix.

PROOF. Evaluate the left-hand side using (35) and then (33), (34). Simplify the result using (29)-(31) and $A_{1}^{\varepsilon}=-\mathbf{i}\left(A_{1} A_{1}^{*}-A_{1}^{*} A_{1}\right) / 2$. 


\section{The eigenvalues of the imaginary adjacency matrix}

In this section we describe the eigenvalues for the imaginary adjacency matrix of $Q_{D}$. We begin with a definition.

Definition 7.1 With reference to Notation 6.1 we define

$$
P=P_{1}^{\otimes D}
$$

where $P_{1}$ is the matrix with rows and columns indexed by the set $\{0,1\}$, and with $(0,0)$-entry $1,(0,1)$-entry $1,(1,0)$-entry $-\mathbf{i}$, and $(1,1)$-entry $\mathbf{i}$.

Lemma 7.2 With reference to Notation 6.1 and Definition 7.1 we have $P \in T$.

PROOF. Observe that the symmetric group $S_{D}$ acts on $X$ as a group of automorphisms of $Q_{D}$ by the rule

$$
\left(t_{1}, t_{2}, \ldots, t_{D}\right)^{\sigma}=\left(t_{\sigma(1)}, t_{\sigma(2)}, \ldots, t_{\sigma(D)}\right) \quad\left(\sigma \in S_{D}\right),
$$

where $\left(t_{1}, t_{2}, \ldots, t_{D}\right)$ is a vertex of $Q_{D}$. In fact $S_{D}$ is isomorphic to the stabilizer of the vertex $x$ in the full automorphism group of $Q_{D}$ [3, Theorem 9.2.1].

Observe that the above $S_{D}$-action on $X$ induces an $S_{D}$-action on $V$. For $\sigma \in S_{D}$ let $M_{\sigma}$ denote the matrix in $\operatorname{Mat}_{X}(\mathbb{C})$ that represents $\sigma$ with respect to the basis $\{\hat{y} \mid y \in X\}$. By [16, Subsection I.C] $T$ is the centralizer algebra for $S_{D}$ on $V$, i.e.

$$
T=\left\{M \in \operatorname{Mat}_{X}(\mathbb{C}) \mid M M_{\sigma}=M_{\sigma} M \forall \sigma \in S_{D}\right\}
$$

Pick distinct $1 \leq i, j \leq D$ and consider the involution $\sigma=(i, j) \in S_{D}$. Since $S_{D}$ is generated by the involutions, to show $P \in T$ it suffices to show $P M_{\sigma}=M_{\sigma} P$. For $y, z \in X$ the $(y, z)$-entry of $M_{\sigma}$ is 1 if $y^{\sigma}=z$ and 0 if $y^{\sigma} \neq z$. By this and matrix multiplication the $(y, z)$-entry of $P M_{\sigma}$ is $P_{y z^{\sigma}}$ and the $(y, z)$-entry of $M_{\sigma} P$ is $P_{y^{\sigma}} z$. By (37) and the definition of the Kronecker product $P_{y z^{\sigma}}=P_{y^{\sigma}}$. By these comments $\left(P M_{\sigma}\right)_{y z}=\left(M_{\sigma} P\right)_{y z}$. Therefore $P M_{\sigma}=M_{\sigma} P$ so $P \in T$.

We have an observation.

Lemma 7.3 With reference to Notation 6.1 and Definition 7.1 the following (i)-(iii) hold.

(i) $P \bar{P}^{t}=\bar{P}^{t} P=2^{D} I$;

(ii) $P^{3}=2^{D}(1-\mathbf{i})^{D} I$;

(iii) $P^{-1}$ exists.

Proof. (i) We first observe that $P_{1}{\overline{P_{1}}}^{t}=2 I_{1}$, where $I_{1}$ denotes the $2 \times 2$ identity matrix. Using (29) and (32) we now obtain

$$
P \bar{P}^{t}=P_{1}^{\otimes D}\left({\overline{P_{1}}}^{t}\right)^{\otimes D}=\left(P_{1}{\overline{P_{1}}}^{t}\right)^{\otimes D}=\left(2 I_{1}\right)^{\otimes D}=2^{D} I .
$$


(ii) We first observe that $P_{1}^{3}=2(1-\mathbf{i}) I_{1}$. Using (29) we now obtain

$$
P^{3}=P_{1}^{\otimes D} P_{1}^{\otimes D} P_{1}^{\otimes D}=\left(P_{1}^{3}\right)^{\otimes D}=\left(2(1-\mathbf{i}) I_{1}\right)^{\otimes D}=2^{D}(1-\mathbf{i})^{D} I .
$$

(iii) Clear from (ii) above.

Theorem 7.4 With reference to Notation 6.1 and Definition 7.1,

$$
A^{*}=P A P^{-1}, \quad A^{\varepsilon}=P A^{*} P^{-1}, \quad A=P A^{\varepsilon} P^{-1} .
$$

Proof. To verify the equation on the left, evaluate $A^{*}$ using (34) and $P A P^{-1}$ using (29), (33), (37). Comparing the results using $A_{1}^{*}=P_{1} A_{1} P_{1}^{-1}$ we find $A^{*}=P A P^{-1}$. The other two equations are similarly obtained.

Corollary 7.5 With reference to Notation 6.1 the following (i)-(iii) hold.

(i) The matrix $A^{\varepsilon}$ is diagonalizable.

(ii) The eigenvalues of $A^{\varepsilon}$ are $D-2 i(0 \leq i \leq D)$.

(iii) For $0 \leq i \leq D$ the eigenvalue $D-2 i$ of $\overline{A^{\varepsilon}}$ has multiplicity $\left(\begin{array}{c}D \\ i\end{array}\right)$.

PROOF. Use (23) and the equation on the right in Theorem 7.4.

\section{The primitive idempotents of the imaginary adjacency matrix}

In this section we consider the primitive idempotents for the imaginary adjacency matrix of $Q_{D}$. We start with a definition.

Definition 8.1 With reference to Notation 6.1, for $0 \leq i \leq D$ we define $E_{i}^{\varepsilon}=$ $P^{-1} E_{i} P$, where $P$ is from Definition 7.1 and $E_{i}$ is the $i$ th primitive idempotent of $Q_{D}$.

Adopt Notation 6.1. Using Definition 8.1, (3)-(7), (9), Lemma 7.3(i), and the equation on the right in Theorem 7.4 we routinely find

$$
\begin{array}{r}
E_{0}^{\varepsilon}+E_{1}^{\varepsilon}+\cdots+E_{D}^{\varepsilon}=I, \\
{\overline{E_{i}^{\varepsilon}}}^{t}=E_{i}^{\varepsilon}(0 \leq i \leq D), \\
E_{i}^{\varepsilon} E_{j}^{\varepsilon}=\delta_{i j} E_{i}^{\varepsilon}(0 \leq i, j \leq D), \\
A^{\varepsilon}=\sum_{i=0}^{D}(D-2 i) E_{i}^{\varepsilon}, \\
A^{\varepsilon} E_{i}^{\varepsilon}=E_{i}^{\varepsilon} A^{\varepsilon}=(D-2 i) E_{i}^{\varepsilon}(0 \leq i \leq D), \\
E_{i}^{\varepsilon}=\prod_{\substack{0 \leq j \leq D \\
j \neq i}} \frac{A^{\varepsilon}-\theta_{j} I}{\theta_{i}-\theta_{j}}(0 \leq i \leq D) .
\end{array}
$$


For $0 \leq i \leq D$ we note that $E_{i}^{\varepsilon}$ is the primitive idempotent of $A^{\varepsilon}$ associated with the eigenvalue $D-2 i$. It follows from (44) that $E_{i}^{\varepsilon} \in T$. We call $E_{i}^{\varepsilon}$ the $i$ th imaginary idempotent of $Q_{D}$ with respect to $x$. For the rest of this paper we consider the following situation.

Notation 8.2 Let $D$ denote a positive integer and let $Q_{D}=(X, R)$ denote the $D$ cube. Let $A$ denote the adjacency matrix of $Q_{D}$ and let $I$ denote the identity matrix in $\operatorname{Mat}_{X}(\mathbb{C})$. Let $V$ denote the standard module of $\operatorname{Mat}_{X}(\mathbb{C})$. Fix $x=(0,0, \ldots, 0) \in X$, and let $A^{*}=A^{*}(x)$ and $A^{\varepsilon}=A^{\varepsilon}(x)$ denote the corresponding dual adjacency and the imaginary adjacency matrix, respectively. Let $T=T(x)$ denote the corresponding Terwilliger algebra. Let $E_{i}, E_{i}^{*}, E_{i}^{\varepsilon}(0 \leq i \leq D)$ denote the primitive idempotents, the dual idempotents and the imaginary idempotents of $Q_{D}$, respectively. Let the matrix $P$ be as in Definition 7.1.

We record the following for later use.

Lemma 8.3 With reference to Notation 8.2 the following (i), (ii) hold.

(i) For $0 \leq i \leq D, E_{i}^{\varepsilon} V$ is the eigenspace of $A^{\varepsilon}$ for the eigenvalue $D-2 i$.

(ii) $V=E_{0}^{\varepsilon} V+E_{1}^{\varepsilon} V+\cdots+E_{D}^{\varepsilon} V$ (orthogonal direct sum).

ProOF. (i) This follows from (43).

(ii) Evaluate $V=P^{-1} I P V$ using (3) and Definition 8.1 to obtain $V=\sum_{i=0}^{D} E_{i}^{\varepsilon} V$ (direct sum). This sum is orthogonal by (40) and (41).

Lemma 8.4 With reference to Notation 8.2 the following holds for $0 \leq i \leq D$ :

$$
E_{i}^{*}=P E_{i} P^{-1}, \quad E_{i}^{\varepsilon}=P E_{i}^{*} P^{-1}, \quad E_{i}=P E_{i}^{\varepsilon} P^{-1} .
$$

PROOF. To verify the left equation, simplify the right-hand side using (9) and the equation on the left in Theorem 7.4. Compare the result with (17) and recall $\theta_{i}=\theta_{i}^{*}$ for $0 \leq i \leq D$. The other two equations are similarly obtained.

Corollary 8.5 With reference to Notation 8.2, let $W$ denote an irreducible T-module. Then the following holds for $0 \leq i \leq D$ :

$$
P E_{i} W=E_{i}^{*} W, \quad P E_{i}^{*} W=E_{i}^{\varepsilon} W, \quad P E_{i}^{\varepsilon} W=E_{i} W .
$$

PROOF. Immediate from Lemma 8.4 and since $P^{-1} W=W$ by Lemma 7.2.

Corollary 8.6 With reference to Notation 8.2, let $W$ denote an irreducible $T$-module. Then $\operatorname{dim}\left(E_{i}^{\varepsilon} W\right) \leq 1$ for $0 \leq i \leq D$.

PROOF. This follows from Corollary 8.5 and since $\operatorname{dim}\left(E_{i}^{*} W\right) \leq 1$ for $0 \leq i \leq D$.

Lemma 8.7 With reference to Notation 8.2, let $W$ denote an irreducible T-module with endpoint $r$ and diameter $d=D-2 r$. Then the following (i), (ii) hold. 
(i) $E_{i}^{\varepsilon} W \neq 0$ if and only if $r \leq i \leq r+d(0 \leq i \leq D)$;

(ii) $W=E_{r}^{\varepsilon} W+E_{r+1}^{\varepsilon} W+\cdots+E_{r+d}^{\varepsilon} W$ (orthogonal direct sum).

Proof. (i) By Corollary 8.5 we have $E_{i}^{\varepsilon} W=P E_{i}^{*} W$. By (21) we have $E_{i}^{*} W \neq 0$ if and only if $r \leq i \leq r+d$. The result follows.

(ii) Recall that $W=\sum_{i=0}^{d} E_{r+i}^{*} W$ (direct sum) by (21). Therefore $P W=$ $\sum_{i=0}^{d} P E_{r+i}^{*} W$ (direct sum). Simplify this equation using $W=P W$ and Corollary 8.5 to obtain $W=\sum_{i=0}^{d} E_{r+i}^{\varepsilon} W$ (direct sum). This sum is orthogonal by (40) and (41).

Lemma 8.8 With reference to Notation 8.2 the following (i)-(v) are equivalent for $0 \leq h, j \leq D$ :

(i) $p_{1 j}^{h}=0$;

(ii) $E_{h} A^{\varepsilon} E_{j}=0$;

(iii) $E_{h}^{*} A^{\varepsilon} E_{j}^{*}=0$;

(iv) $E_{h}^{\varepsilon} A E_{j}^{\varepsilon}=0$;

(v) $E_{h}^{\varepsilon} A^{*} E_{j}^{\varepsilon}=0$.

Proof. By Theorem 7.4 and Lemma 8.4 we have $P E_{h}^{*} A E_{j}^{*} P^{-1}=E_{h}^{\varepsilon} A^{*} E_{j}^{\varepsilon}$, $P^{2} E_{h}^{*} A E_{j}^{*} P^{-2}=E_{h} A^{\varepsilon} E_{j}, P E_{h} A^{*} E_{j} P^{-1}=E_{h}^{*} A^{\varepsilon} E_{j}^{*}$ and $P^{2} E_{h} A^{*} E_{j} P^{-2}=$ $E_{h}^{\varepsilon} A E_{j}^{\varepsilon}$. The result now follows in view of (18), (19) and (24).

\section{Six bases for an irreducible $T$-module}

With reference to Notation 8.2, let $W$ denote an irreducible $T$-module. We are going to show that the triple $A, A^{*}, A^{\varepsilon}$ acts on $W$ as a Leonard triple. We start with a lemma.

Lemma 9.1 With reference to Notation 8.2, let $W$ denote an irreducible T-module with endpoint $r$ and diameter $d=D-2 r$. Then the following (i)-(iii) hold.

(i) For a nonzero $u \in E_{r} W$, each of the following two sequences is a basis for $W$ :

$$
\begin{aligned}
& E_{r}^{*} u, E_{r+1}^{*} u, \ldots, E_{r+d}^{*} u ; \\
& E_{r}^{\varepsilon} u, E_{r+1}^{\varepsilon} u, \ldots, E_{r+d}^{\varepsilon} u .
\end{aligned}
$$

(ii) For a nonzero $u^{*} \in E_{r}^{*} W$, each of the following two sequences is a basis for $W$ :

$$
\begin{aligned}
& E_{r}^{\varepsilon} u^{*}, E_{r+1}^{\varepsilon} u^{*}, \ldots, E_{r+d}^{\varepsilon} u^{*} ; \\
& E_{r} u^{*}, E_{r+1} u^{*}, \ldots, E_{r+d} u^{*} .
\end{aligned}
$$


(iii) For a nonzero $u^{\varepsilon} \in E_{r}^{\varepsilon} W$, each of the following two sequences is a basis for $W$ :

$$
\begin{aligned}
& E_{r} u^{\varepsilon}, E_{r+1} u^{\varepsilon}, \ldots, E_{r+d} u^{\varepsilon} ; \\
& E_{r}^{*} u^{\varepsilon}, E_{r+1}^{*} u^{\varepsilon}, \ldots, E_{r+d}^{*} u^{\varepsilon} .
\end{aligned}
$$

PROOF. It follows from [10, Corollaries 6.8 and 8.5] that the pair $A, A^{*}$ acts on $W$ as a Leonard pair. Therefore (45) and (48) are bases for $W$ by [24, Lemma 10.2].

By Lemma 8.4 and Corollary 8.5 the sequence (47) (resp. (49)) is the image under $P$ (resp. $P^{2}$ ) of the sequence (45), provided $u$ is normalized so that $P u=u^{*}$ (resp. $P^{2} u=u^{\varepsilon}$ ). Similarly, the sequence (50) (resp. (46)) is the image under $P$ (resp. $P^{2}$ ) of the sequence (48), provided $u^{*}$ is normalized so that $P u^{*}=u^{\varepsilon}\left(\right.$ resp. $\left.P^{2} u^{*}=u\right)$. Since $P$ is invertible, the sequences (46), (47), (49) and (50) are bases for $W$.

The following result will be useful.

Lemma 9.2 With reference to Notation 8.2, let $W$ denote an irreducible T-module with endpoint $r$ and diameter $d=D-2 r$. Then the following (i)-(iii) hold.

(i) For a nonzero $u \in E_{r} W$,

$$
u=\sum_{i=0}^{d} E_{r+i}^{*} u, \quad u=\sum_{i=0}^{d} E_{r+i}^{\varepsilon} u .
$$

(ii) For a nonzero $u^{*} \in E_{r}^{*} W$,

$$
u^{*}=\sum_{i=0}^{d} E_{r+i}^{\varepsilon} u^{*}, \quad u^{*}=\sum_{i=0}^{d} E_{r+i} u^{*} .
$$

(iii) For a nonzero $u^{\varepsilon} \in E_{r}^{\varepsilon} W$,

$$
u^{\varepsilon}=\sum_{i=0}^{d} E_{r+i} u^{\varepsilon}, \quad u^{\varepsilon}=\sum_{i=0}^{d} E_{r+i}^{*} u^{\varepsilon} .
$$

ProOF. (i) Evaluate $u=I u$ using (10) and (21) to obtain the equation on the left in (51). Evaluate $u=I u$ using (39) and Lemma 8.7(i) to obtain the equation on the right in (51).

(ii), (iii) Similar to the proof of (i) above.

For future use we record an idea from the proof of Lemma 9.1.

Lemma 9.3 With reference to Notation 8.2, let $W$ denote an irreducible T-module with endpoint $r$ and diameter $d=D-2 r$. Then the following (i), (ii) hold.

(i) The basis (47) (resp. (49), (45)) is the image under $P$ of the basis (45) (resp. (47), (49)), provided $u$ (resp. $\left.u^{*}, u^{\varepsilon}\right)$ is normalized so that $P u=u^{*}$ (resp. $P u^{*}=u^{\varepsilon}$, $\left.P u^{\varepsilon}=u\right)$. 
(ii) The basis (48) (resp. (50), (46)) is the image under $P$ of the basis (46) (resp. (48), (50)), provided $u$ (resp. $\left.u^{*}, u^{\varepsilon}\right)$ is normalized so that $P u=u^{*}$ (resp. $P u^{*}=u^{\varepsilon}$, $\left.P u^{\varepsilon}=u\right)$.

Remark 9.4 With reference to Notation 8.2, let $W$ denote an irreducible $T$-module with endpoint $r$ and diameter $d=D-2 r$. Note that the definition of a standard basis for $W$ [10, Definition 6.4] is different from the definition of the basis (45). However, it turns out that these definitions are equivalent. Similarly, the definition of a dual standard basis for $W$ [10, Definition 8.2] is equivalent to the definition of the basis (48). We will therefore prove all the results of Section 10 and Section 11, although some of these results were already proven in [10].

\section{The action of $A, A^{*}, A^{\varepsilon}$ on the six bases}

With reference to Notation 8.2 , let $W$ denote an irreducible $T$-module with diameter $d$. In this section we display the matrices which represent the action of $A, A^{*}$ and $A^{\varepsilon}$ on $W$ with respect to the six bases from Lemma 9.1. We use the following notation. Let $\operatorname{Mat}_{d+1}(\mathbb{C})$ denote the $\mathbb{C}$-algebra of all $(d+1) \times(d+1)$ matrices with entries in $\mathbb{C}$. The rows and columns of matrices in $\operatorname{Mat}_{d+1}(\mathbb{C})$ shall be indexed by $0,1, \ldots, d$. Let $v_{0}, v_{1}, \ldots, v_{d}$ denote a basis for $W$. For $B \in \operatorname{Mat}_{d+1}(\mathbb{C})$ and $Y \in T$ we say $B$ represents $Y$ with respect to $v_{0}, v_{1}, \ldots, v_{d}$ whenever $Y v_{j}=\sum_{i=0}^{d} B_{i j} v_{i}$ for $0 \leq j \leq d$. We have a comment. For an invertible $S \in T$ the following are equivalent: (i) the matrix $B$ represents $Y$ with respect to $v_{0}, v_{1}, \ldots, v_{d}$; (ii) the matrix $B$ represents $S Y S^{-1}$ with respect to $S v_{0}, S v_{1}, \ldots, S v_{d}$.

Theorem 10.1 With reference to Notation 8.2, let $W$ denote an irreducible T-module with endpoint $r$ and diameter $d=D-2 r$. Then the following (i)-(iii) hold.

(i) The matrix which represents $A$ with respect to the basis (48) of $W$ and with respect to the basis (49) of $W$ is $\operatorname{diag}(d, d-2, d-4, \ldots,-d)$.

(ii) The matrix which represents $A^{*}$ with respect to the basis (45) of $W$ and with respect to the basis (50) of $W$ is $\operatorname{diag}(d, d-2, d-4, \ldots,-d)$.

(iii) The matrix which represents $A^{\varepsilon}$ with respect to the basis (46) of $W$ and with respect to the basis (47) of $W$ is $\operatorname{diag}(d, d-2, d-4, \ldots,-d)$.

PROOF. (i) Recall that the basis (48) of $W$ is of the form $E_{r} u^{*}, E_{r+1} u^{*}, \ldots, E_{r+d} u^{*}$, where $u^{*}$ is a nonzero vector in $E_{r}^{*} W$. Similarly, the basis (49) of $W$ is of the form $E_{r} u^{\varepsilon}, E_{r+1} u^{\varepsilon}, \ldots, E_{r+d} u^{\varepsilon}$, where $u^{\varepsilon}$ is a nonzero vector in $E_{r}^{\varepsilon} W$. For $0 \leq i \leq d$ each of $E_{r+i} u^{*}, E_{r+i} u^{\varepsilon}$ is an eigenvector for $A$ with eigenvalue $\theta_{r+i}=d-2 i$. The result follows.

(ii), (iii) Similar to the proof of (i) above.

Theorem 10.2 With reference to Notation 8.2, let $W$ denote an irreducible $T$ module with endpoint $r$ and diameter $d=D-2 r$. Consider the following matrix 
in $\operatorname{Mat}_{d+1}(\mathbb{C})$ :

$$
\left(\begin{array}{ccccc}
0 & d & & & 0 \\
1 & 0 & d-1 & & \\
2 & \cdot & \cdot & \\
& & \cdot & \cdots & \\
& & & \cdots & 1 \\
0 & & & d & 0
\end{array}\right)
$$

Then the following (i)-(iii) hold.

(i) The matrix (54) represents $A$ with respect to the bases (45) and (46) of $W$.

(ii) The matrix (54) represents $A^{*}$ with respect to the bases (47) and (48) of $W$.

(iii) The matrix (54) represents $A^{\varepsilon}$ with respect to the bases (49) and (50) of W.

Proof. (i) Recall that the basis (45) is of the form $E_{r}^{*} u, E_{r+1}^{*} u, \ldots, E_{r+d}^{*} u$ where $u$ is a nonzero vector in $E_{r} W$. Let $B$ denote the matrix in $\operatorname{Mat}_{d+1}(\mathbb{C})$ which represents $A$ with respect to this basis. We show that $B$ is equal to the matrix (54).

For $0 \leq i \leq D$ we have $A E_{i}^{*} V \subseteq E_{i-1}^{*} V+E_{i+1}^{*} V$ by (18) and (25); therefore $A E_{i}^{*} W \subseteq E_{i-1}^{*} W+E_{i+1}^{*} W$, implying that $B$ is tridiagonal with diagonal entries 0 . Further, $(A-d I) u=0$ since $u \in E_{r} W$ and $\theta_{r}=d$. Therefore $(B-$ $d I)(1,1, \ldots, 1)^{t}=0$ by the equation on the left in (51). By these comments

$$
B_{0,1}=d, \quad B_{i, i-1}+B_{i, i+1}=d \quad(1 \leq i \leq d-1), \quad B_{d, d-1}=d .
$$

By (19), (24) and (25) we find $A^{*} E_{r} V \subseteq E_{r-1} V+E_{r+1} V$. By this and since $W$ has endpoint $r$ we find $A^{*} E_{r} W \subseteq E_{r+1} W$. Therefore $A^{*} u \in E_{r+1} W$. Now $(A-(d-$ 2)I) $A^{*} u=0$ since $\theta_{r+1}=d-2$. As $A^{*} u=\sum_{i=0}^{d} \theta_{r+i} E_{r+i}^{*} u$ by construction, this implies $(B-(d-2) I)\left(\theta_{r}, \theta_{r+1}, \ldots, \theta_{r+d}\right)^{t}=0$. Combining this with the fact that $B$ is tridiagonal with diagonal entries 0 we find

$$
B_{i, i-1} \theta_{r+i-1}+B_{i, i+1} \theta_{r+i+1}=(d-2) \theta_{r+i} \quad(1 \leq i \leq d-1) .
$$

Combining (55) and (56) and using $\theta_{r+j}=d-2 j$ for $0 \leq j \leq d$ we obtain $B_{i, i-1}=i$ for $1 \leq i \leq d$ and $B_{i, i+1}=d-i$ for $0 \leq i \leq d-1$. Therefore $B$ is equal to the matrix (54).

Next recall that the basis (46) is of the form $E_{r}^{\varepsilon} u, E_{r+1}^{\varepsilon} u, \ldots, E_{r+d}^{\varepsilon} u$ where $u$ is a nonzero vector in $E_{r} W$. Let $B^{\prime}$ denote the matrix in $\operatorname{Mat}_{d+1}(\mathbb{C})$ which represents $A$ with respect to this basis. We show that $B^{\prime}$ is equal to the matrix (54). Since the proof is similar to the proof that $B$ is equal to the matrix (54), we just indicate the main steps.

Similarly as in the first part of the proof, but using Lemma 8.8 instead of (18), we find that $B^{\prime}$ is tridiagonal with diagonal entries 0 . Since $(A-d I) u=0$ we obtain $\left(B^{\prime}-d I\right)(1,1, \ldots, 1)^{t}=0$ by the equation on the right in $(51)$. Hence

$$
B_{0,1}^{\prime}=d, \quad B_{i, i-1}^{\prime}+B_{i, i+1}^{\prime}=d \quad(1 \leq i \leq d-1), \quad B_{d, d-1}^{\prime}=d .
$$

Further, $A^{\varepsilon} E_{r} W \subseteq E_{r+1} W$ by Lemma 8.8 and (25), implying $(A-(d-2) I) \times$ $A^{\varepsilon} u=0$. This gives us $\left(B^{\prime}-(d-2) I\right)\left(\theta_{r}, \theta_{r+1}, \ldots, \theta_{r+d}\right)^{t}=0$ since $A^{\varepsilon} u=$ 
$\sum_{i=0}^{d} \theta_{r+i} E_{r+i}^{\varepsilon} u$. Hence

$$
B_{i, i-1}^{\prime} \theta_{r+i-1}+B_{i, i+1}^{\prime} \theta_{r+i+1}=(d-2) \theta_{r+i} \quad(1 \leq i \leq d-1) .
$$

Combining (57) and (58) we find that $B^{\prime}$ is equal to the matrix (54).

(ii), (iii) Use Theorem 7.4, Lemma 9.3 and the comment above Theorem 10.1.

Theorem 10.3 With reference to Notation 8.2, let $W$ denote an irreducible $T$ module with endpoint $r$ and diameter $d=D-2 r$. Consider the following matrix in $\operatorname{Mat}_{d+1}(\mathbb{C})$ :

$$
\mathbf{i}\left(\begin{array}{cccccc}
0 & d & & & & 0 \\
-1 & 0 & d-1 & & \\
& -2 & \cdot & \cdot & & \\
& & \cdot & \cdot & \cdot & \\
& & & \cdot & \cdot & 1 \\
0 & & & & -d & 0
\end{array}\right) .
$$

Then the following (i)-(iii) hold.

(i) The matrix (59) represents $A$ with respect to the basis (47) of $W$.

(ii) The matrix (59) represents $A^{*}$ with respect to the basis (49) of $W$.

(iii) The matrix (59) represents $A^{\varepsilon}$ with respect to the basis (45) of $W$.

Proof. (i) Let $B, B^{*}$ and $B^{\varepsilon}$ denote the matrices in Mat $d+1(\mathbb{C})$ which represent $A$, $A^{*}$ and $A^{\varepsilon}$ with respect to the basis (47) of $W$. We have $B=-\mathbf{i}\left(B^{*} B^{\varepsilon}-B^{\varepsilon} B^{*}\right) / 2$ by Lemma 6.3(ii). Recall that $B^{\varepsilon}$ is equal to $\operatorname{diag}(d, d-2, d-4, \ldots,-d)$ by Theorem 10.1(iii) and that $B^{*}$ is equal to the matrix (54) by Theorem 10.2(ii). By these comments $B$ is equal to the matrix (59).

(ii), (iii) Use Theorem 7.4, Lemma 9.3 and the comment above Theorem 10.1.

Theorem 10.4 With reference to Notation 8.2, let $W$ denote an irreducible $T$ module with endpoint $r$ and diameter $d=D-2 r$. Consider the following matrix in $\operatorname{Mat}_{d+1}(\mathbb{C})$ :

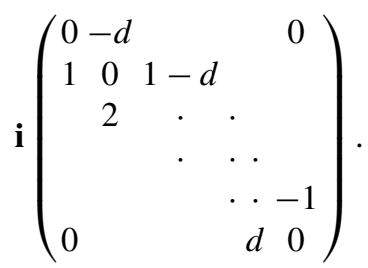

Then the following (i)-(iii) hold.

(i) The matrix (60) represents $A$ with respect to the basis (50) of $W$.

(ii) The matrix (60) represents $A^{*}$ with respect to the basis (46) of $W$.

(iii) The matrix (60) represents $A^{\varepsilon}$ with respect to the basis (48) of $W$. 
Proof. (i) Let $B, B^{*}$ and $B^{\varepsilon}$ denote the matrices in Mat $d+1(\mathbb{C})$ which represent $A$, $A^{*}$ and $A^{\varepsilon}$ with respect to the basis (50) of $W$. We have $B=-\mathbf{i}\left(B^{*} B^{\varepsilon}-B^{\varepsilon} B^{*}\right) / 2$ by Lemma 6.3 (ii). Recall that $B^{*}$ is equal to $\operatorname{diag}(d, d-2, d-4, \ldots,-d)$ by Theorem 10.1(ii) and that $B^{\varepsilon}$ is equal to the matrix (54) by Theorem 10.2(iii). By these comments $B$ is equal to the matrix (60).

(ii), (iii) Use Theorem 7.4, Lemma 9.3 and the comment above Theorem 10.1.

Corollary 10.5 With reference to Notation 8.2, let $W$ denote an irreducible $T$ module. Then the triple $A, A^{*}, A^{\varepsilon}$ acts on $W$ as a Leonard triple.

PROOF. Immediate from Theorems 10.1 - 10.4.

\section{The inner products}

With reference to Notation 8.2, let $W$ denote an irreducible $T$-module. In Lemma 9.1 we displayed six bases for $W$. Later in the paper we will find the transition matrices between these bases. Before we get to this it is convenient to find the inner products for the vectors in these bases.

Theorem 11.1 With reference to Notation 8.2, let $W$ denote an irreducible T-module with endpoint $r$ and diameter $d=D-2 r$. Then the following (i)-(iii) hold for $0 \leq$ $i, j \leq d$.

(i) For a nonzero $u \in E_{r} W$,

$$
\left\langle E_{r+i}^{*} u, E_{r+j}^{*} u\right\rangle=\delta_{i j}\left(\begin{array}{c}
d \\
i
\end{array}\right) 2^{-d}\|u\|^{2}, \quad\left\langle E_{r+i}^{\varepsilon} u, E_{r+j}^{\varepsilon} u\right\rangle=\delta_{i j}\left(\begin{array}{c}
d \\
i
\end{array}\right) 2^{-d}\|u\|^{2} .
$$

(ii) For a nonzero $u^{*} \in E_{r}^{*} W$,

$$
\left\langle E_{r+i}^{\varepsilon} u^{*}, E_{r+j}^{\varepsilon} u^{*}\right\rangle=\delta_{i j}\left(\begin{array}{l}
d \\
i
\end{array}\right) 2^{-d}\left\|u^{*}\right\|^{2}, \quad\left\langle E_{r+i} u^{*}, E_{r+j} u^{*}\right\rangle=\delta_{i j}\left(\begin{array}{c}
d \\
i
\end{array}\right) 2^{-d}\left\|u^{*}\right\|^{2} .
$$

(iii) For a nonzero $u^{\varepsilon} \in E_{r}^{\varepsilon} W$,

$$
\left\langle E_{r+i} u^{\varepsilon}, E_{r+j} u^{\varepsilon}\right\rangle=\delta_{i j}\left(\begin{array}{c}
d \\
i
\end{array}\right) 2^{-d}\left\|u^{\varepsilon}\right\|^{2}, \quad\left\langle E_{r+i}^{*} u^{\varepsilon}, E_{r+j}^{*} u^{\varepsilon}\right\rangle=\delta_{i j}\left(\begin{array}{c}
d \\
i
\end{array}\right) 2^{-d}\left\|u^{\varepsilon}\right\|^{2} .
$$

PROOF. (i) Concerning the equation on the left, it holds for $i \neq j$ since $E_{r+i}^{*} u$ and $E_{r+j}^{*} u$ are orthogonal by (16). To verify the equation for $i=j$ we first claim that $\left\|E_{r+i}^{*} u\right\|^{2}=\left(\begin{array}{c}d \\ i\end{array}\right)\left\|E_{r}^{*} u\right\|^{2}$. To prove the claim we assume $i \geq 1$; otherwise the result is 
clear. By (1) and since $\bar{A}^{t}=A$ we have

$$
\left\langle A E_{r+i-1}^{*} u, E_{r+i}^{*} u\right\rangle=\left\langle E_{r+i-1}^{*} u, A E_{r+i}^{*} u\right\rangle .
$$

In this equation we evaluate both sides using Theorem 10.2(i) and simplify the result using the fact that $E_{r}^{*} u, E_{r+1}^{*} u, \ldots, E_{r+d}^{*} u$ are mutually orthogonal; we obtain

$$
i\left\|E_{r+i}^{*} u\right\|^{2}=(d-i+1)\left\|E_{r+i-1}^{*} u\right\|^{2} .
$$

The claim follows from this and induction on $i$. Next we claim that $\left\|E_{r}^{*} u\right\|^{2}=$ $2^{-d}\|u\|^{2}$. To see this, recall that $E_{r}^{*} u, E_{r+1}^{*} u, \ldots, E_{r+d}^{*} u$ are mutually orthogonal and that $u=\sum_{i=0}^{d} E_{r+i}^{*} u$ by the equation on the left in Lemma 9.2(i). By these comments and the first claim,

$$
\|u\|^{2}=\sum_{i=0}^{d}\left\|E_{r+i}^{*} u\right\|^{2}=\left\|E_{r}^{*} u\right\|^{2} \sum_{i=0}^{d}\left(\begin{array}{l}
d \\
i
\end{array}\right)=2^{d}\left\|E_{r}^{*} u\right\|^{2}
$$

and the second claim is proved. Combining the two claims we get the equation on the left for $i=j$. We have now verified the equation on the left. The proof for the equation on the right is similar, so we just indicate the main steps. If $i \neq j$ then $E_{r+i}^{\varepsilon} u$ and $E_{r+j}^{\varepsilon} u$ are orthogonal by Lemma 8.3(ii). Assume now $i=j$. By (1) we have $\left\langle A E_{r+i-1}^{\varepsilon} u, E_{r+i}^{\varepsilon} u\right\rangle=\left\langle E_{r+i-1}^{\varepsilon} u, A E_{r+i}^{\varepsilon} u\right\rangle$. Evaluating and simplifying this using Theorem 10.2(i) and the fact that $E_{r}^{\varepsilon} u, E_{r+1}^{\varepsilon} u, \ldots, E_{r+d}^{\varepsilon} u$ are mutually orthogonal we obtain $i\left\|E_{r+i}^{\varepsilon} u\right\|^{2}=(d-i+1)\left\|E_{r+i-1}^{\varepsilon} u\right\|^{2}$. Using induction on $i$ we find $\left\|E_{r+i}^{\varepsilon} u\right\|^{2}=\left(\begin{array}{c}d \\ i\end{array}\right)\left\|E_{r}^{\varepsilon} u\right\|^{2}$. Using this and the equation on the right in Lemma 9.2(i) we find $\|u\|^{2}=2^{d}\left\|E_{r}^{\varepsilon} u\right\|^{2}$. Combining the above results we get the equation on the right.

(ii), (iii) Use (1), Lemma 7.3(i), Lemma 9.3 and (i) above.

We have a comment.

Theorem 11.2 With reference to Notation 8.2, let $W$ denote an irreducible $T$-module with endpoint $r$ and diameter $d=D-2 r$. Then the following (i)-(iii) hold for $0 \leq$ $i \leq d$ and nonzero vectors $u \in E_{r} W, u^{*} \in E_{r}^{*} W, u^{\varepsilon} \in E_{r}^{\mathcal{E}} W$.

(i) $E_{r+i} u^{\varepsilon}=\mathbf{i}^{i}(1-\mathbf{i})^{d}\left\langle u^{\varepsilon}, u^{*}\right\rangle\left\|u^{*}\right\|^{-2} E_{r+i} u^{*}$.

(ii) $E_{r+i}^{*} u=\mathbf{i}^{i}(1-\mathbf{i})^{d}\left\langle u, u^{\varepsilon}\right\rangle\left\|u^{\varepsilon}\right\|^{-2} E_{r+i}^{*} u^{\varepsilon}$.

(iii) $E_{r+i}^{\varepsilon} u^{*}=\mathbf{i}^{i}(1-\mathbf{i})^{d}\left\langle u^{*}, u\right\rangle\|u\|^{-2} E_{r+i}^{\varepsilon} u$.

PROOF. (i) Each of $E_{r+i} u^{\varepsilon}, E_{r+i} u^{*}$ is a basis for $E_{r+i} W$ so there exists a nonzero $\lambda_{i} \in \mathbb{C}$ such that $E_{r+i} u^{\varepsilon}=\lambda_{i} E_{r+i} u^{*}$. We first claim that $\lambda_{i}=\mathbf{i}^{i} \lambda_{0}$. To prove the claim assume $i \geq 1$; otherwise the result is clear. Note that $A^{*} E_{r+i} u^{\varepsilon}=\lambda_{i} A^{*} E_{r+i} u^{*}$. 
Evaluating both sides of this equation using Theorem 10.2(ii) and Theorem 10.3(ii) and then comparing the results we find that $\lambda_{i}=\mathbf{i} \lambda_{i-1}$. The claim follows from this and induction on $i$. Next we claim that $\lambda_{0}=(1-\mathbf{i})^{d}\left\langle u^{\varepsilon}, u^{*}\right\rangle\left\|u^{*}\right\|^{-2}$. To see this recall that $u^{\varepsilon}=\sum_{i=0}^{d} E_{r+i} u^{\varepsilon}$ and $u^{*}=\sum_{i=0}^{d} E_{r+i} u^{*}$ by Lemma 9.2(ii),(iii). By this, (8), Theorem 11.1(ii) and the first claim we find

$$
\begin{aligned}
\left\langle u^{\varepsilon}, u^{*}\right\rangle & =\sum_{i=0}^{d}\left\langle E_{r+i} u^{\varepsilon}, E_{r+i} u^{*}\right\rangle=\lambda_{0} \sum_{i=0}^{d} \mathbf{i}^{i}\left\|E_{r+i} u^{*}\right\|^{2} \\
& =\frac{\lambda_{0}\left\|u^{*}\right\|^{2}}{2^{d}} \sum_{i=0}^{d}\left(\begin{array}{l}
d \\
i
\end{array}\right) \mathbf{i}^{i}=\frac{\lambda_{0}\left\|u^{*}\right\|^{2}(1+\mathbf{i})^{d}}{2^{d}}
\end{aligned}
$$

and the second claim follows. Combining the two claims we obtain the desired result. (ii), (iii) Use (1), Lemma 7.3(i), Lemma 9.3 and (i) above.

Corollary 11.3 With reference to Notation 8.2, let $W$ denote an irreducible $T$ module with endpoint $r$. Let $u, u^{*}, u^{\varepsilon}$ denote nonzero vectors in $E_{r} W, E_{r}^{*} W, E_{r}^{\varepsilon} W$, respectively. Then each of $\left\langle u^{\varepsilon}, u^{*}\right\rangle,\left\langle u, u^{\varepsilon}\right\rangle,\left\langle u^{*}, u\right\rangle$ is nonzero.

PROOF. The vectors $E_{r} u^{\varepsilon}, E_{r}^{*} u$ and $E_{r}^{\varepsilon} u^{*}$ are nonzero by Lemma 9.1. Combining this with Theorem 11.2 we get the result.

Theorem 11.4 With reference to Notation 8.2, let $W$ denote an irreducible T-module with endpoint $r$ and diameter $d=D-2 r$. Then the following (i)-(iii) hold for $0 \leq$ $i, j \leq d$ and nonzero vectors $u \in E_{r} W, u^{*} \in E_{r}^{*} W, u^{\varepsilon} \in E_{r}^{\varepsilon} W$.

(i) $\left\langle E_{r+i} u^{\varepsilon}, E_{r+j} u^{*}\right\rangle=\delta_{i j} \mathbf{i}^{i}\left(\begin{array}{c}d \\ i\end{array}\right)(1+\mathbf{i})^{-d}\left\langle u^{\varepsilon}, u^{*}\right\rangle$.

(ii) $\left\langle E_{r+i}^{*} u, E_{r+j}^{*} u^{\varepsilon}\right\rangle=\delta_{i j} \mathbf{i}^{i}\left(\begin{array}{c}d \\ i\end{array}\right)(1+\mathbf{i})^{-d}\left\langle u, u^{\varepsilon}\right\rangle$.

(iii) $\left\langle E_{r+i}^{\varepsilon} u^{*}, E_{r+j}^{\varepsilon} u\right\rangle=\delta_{i j} \mathbf{i}^{i}\left(\begin{array}{c}d \\ i\end{array}\right)(1+\mathbf{i})^{-d}\left\langle u^{*}, u\right\rangle$.

PROOF. Immediate from Theorem 11.1 and Theorem 11.2.

Before proceeding we recall a definition. For an integer $n \geq 0$ and $a \in \mathbb{C}$ we define

$$
(a)_{n}=a(a+1)(a+2) \cdots(a+n-1) .
$$

We interpret $(a)_{0}=1$. For integers $0 \leq i, j \leq d$ we define

$$
{ }_{2} F_{1}\left(\begin{array}{c}
-i,-j \\
-d
\end{array} ; 2\right)=\sum_{n=0}^{d} \frac{(-i)_{n}(-j)_{n}}{(-d)_{n} n !} 2^{n} .
$$

The sum (61) is an example of a hypergeometric series [1, Section 2.1]. We will use the fact that

$$
{ }_{2} F_{1}\left(\begin{array}{c}
-i,-j \\
-d
\end{array} ; 2\right)=\frac{d-2 j}{d-i+1}{ }_{2} F_{1}\left(\begin{array}{c}
1-i,-j \\
-d
\end{array} ; 2\right)-\frac{i-1}{d-i+1}{ }_{2} F_{1}\left(\begin{array}{c}
2-i,-j \\
-d
\end{array}{ }^{-2}\right)
$$


provided $i \geq 2$. Line (62) follows from [13, Equation 1.10.3] and since each side of (61) is equal to $K_{i}(j ; 1 / 2, d)\left[13\right.$, Definition 1.10.1] where the $K_{n}(x ; p, N)$ are the Krawtchouk polynomials.

Theorem 11.5 With reference to Notation 8.2, let $W$ denote an irreducible $T$-module with endpoint $r$ and diameter $d=D-2 r$. Then the following (i)-(iii) hold for $0 \leq$ $i, j \leq d$ and nonzero vectors $u \in E_{r} W, u^{*} \in E_{r}^{*} W, u^{\varepsilon} \in E_{r}^{\varepsilon} W$.

(i)

$$
\left\langle E_{r+i} u^{*}, E_{r+j}^{*} u\right\rangle=2^{-d}\left\langle u^{*}, u\right\rangle\left(\begin{array}{l}
d \\
i
\end{array}\right)\left(\begin{array}{l}
d \\
j
\end{array}\right){ }_{2} F_{1}\left(\begin{array}{c}
-i,-j \\
-d
\end{array} ; 2\right) .
$$

(ii)

$$
\left\langle E_{r+i}^{*} u^{\varepsilon}, E_{r+j}^{\varepsilon} u^{*}\right\rangle=2^{-d}\left\langle u^{\varepsilon}, u^{*}\right\rangle\left(\begin{array}{l}
d \\
i
\end{array}\right)\left(\begin{array}{l}
d \\
j
\end{array}\right){ }_{2} F_{1}\left(\begin{array}{c}
-i,-j \\
-d
\end{array} ; 2\right) .
$$

(iii)

$$
\left\langle E_{r+i}^{\varepsilon} u, E_{r+j} u^{\varepsilon}\right\rangle=2^{-d}\left\langle u, u^{\varepsilon}\right\rangle\left(\begin{array}{l}
d \\
i
\end{array}\right)\left(\begin{array}{l}
d \\
j
\end{array}\right){ }_{2} F_{1}\left(\begin{array}{c}
-i,-j \\
-d
\end{array} ; 2\right) .
$$

PROOF. (i) We first claim that

$$
\left\langle E_{r+i} u^{*}, E_{r+j}^{*} u\right\rangle=\left\langle E_{r} u^{*}, E_{r}^{*} u\right\rangle\left(\begin{array}{l}
d \\
i
\end{array}\right)\left(\begin{array}{l}
d \\
j
\end{array}\right){ }_{2} F_{1}\left(\begin{array}{c}
-i,-j \\
-d
\end{array} ; 2\right) .
$$

We will follow the approach of Go [10, Theorem 9.1] and prove the claim using induction on $i+j$. It is clear that (63) holds for $i=j=0$. Assume now $d \geq 1$; otherwise we are done. To show that (63) holds for $(i, j)=(0,1)$ observe by (1), Theorem 10.1(i) and Theorem 10.2(i) that

$$
\left\langle E_{r} u^{*}, E_{r+1}^{*} u\right\rangle=\left\langle E_{r} u^{*}, A E_{r}^{*} u\right\rangle=\left\langle A E_{r} u^{*}, E_{r}^{*} u\right\rangle=d\left\langle E_{r} u^{*}, E_{r}^{*} u\right\rangle .
$$

Similarly (63) holds for $(i, j)=(1,0)$ since

$$
\left\langle E_{r+1} u^{*}, E_{r}^{*} u\right\rangle=\left\langle A^{*} E_{r} u^{*}, E_{r}^{*} u\right\rangle=\left\langle E_{r} u^{*}, A^{*} E_{r}^{*} u\right\rangle=d\left\langle E_{r} u^{*}, E_{r}^{*} u\right\rangle .
$$

To show that (63) holds for $(i, j)=(1,1)$, observe by (1), Theorem 10.1(ii) and Theorem 10.2(ii) that

$$
\left\langle E_{r+1} u^{*}, E_{r+1}^{*} u\right\rangle=\left\langle A^{*} E_{r} u^{*}, E_{r+1}^{*} u\right\rangle=\left\langle E_{r} u^{*}, A^{*} E_{r+1}^{*} u\right\rangle=(d-2)\left\langle E_{r} u^{*}, E_{r+1}^{*} u\right\rangle,
$$

and that the last expression is equal to $d(d-2)\left\langle E_{r} u^{*}, E_{r}^{*} u\right\rangle$ by (64).

For the rest of this proof assume $d \geq 2$ and $i \geq 2$ or $j \geq 2$; otherwise we are done. We first assume $i \geq 2$. By Theorem 10.2(ii),

$$
E_{r+i} u^{*}=\frac{1}{i} A^{*} E_{r+i-1} u^{*}-\frac{d-i+2}{i} E_{r+i-2} u^{*} .
$$


Using this, (1) and Theorem 10.1(ii) we obtain

$$
\left\langle E_{r+i} u^{*}, E_{r+j}^{*} u\right\rangle=\frac{d-2 j}{i}\left\langle E_{r+i-1} u^{*}, E_{r+j}^{*} u\right\rangle-\frac{d-i+2}{i}\left\langle E_{r+i-2} u^{*}, E_{r+j}^{*} u\right\rangle .
$$

By the induction hypothesis the right-hand side of (65) is equal to $\left(\begin{array}{c}d \\ i\end{array}\right)\left(\begin{array}{c}d \\ j\end{array}\right)\left\langle E_{r} u^{*}, E_{r}^{*} u\right\rangle$ times

$$
\frac{d-2 j}{d-i+1}{ }_{2} F_{1}\left(\begin{array}{c}
1-i,-j \\
-d
\end{array} ; 2\right)-\frac{i-1}{d-i+1}{ }_{2} F_{1}\left(\begin{array}{c}
2-i,-j \\
-d
\end{array} ; 2\right) \text {. }
$$

Evaluating the above expression using (62) we obtain (63). Now assume $j \geq 2$. By Theorem 10.2(i),

$$
E_{r+j}^{*} u=\frac{1}{j} A E_{r+j-1}^{*} u-\frac{d-j+2}{j} E_{r+j-2}^{*} u .
$$

Using this, (1) and Theorem 10.1(i) we obtain

$$
\left\langle E_{r+i} u^{*}, E_{r+j}^{*} u\right\rangle=\frac{d-2 i}{j}\left\langle E_{r+i} u^{*}, E_{r+j-1}^{*} u\right\rangle-\frac{d-j+2}{j}\left\langle E_{r+i} u^{*}, E_{r+j-2}^{*} u\right\rangle .
$$

By the induction hypothesis the right-hand side of (66) is equal to $\left(\begin{array}{c}d \\ i\end{array}\right)\left(\begin{array}{c}d \\ j\end{array}\right)\left\langle E_{r} u^{*}, E_{r}^{*} u\right\rangle$ times

$$
\frac{d-2 i}{d-j+1}{ }_{2} F_{1}\left(\begin{array}{c}
-i, 1-j \\
-d
\end{array} ; 2\right)-\frac{j-1}{d-j+1} 2 F_{1}\left(\begin{array}{c}
-i, 2-j \\
-d
\end{array} ; 2\right) .
$$

Evaluating the above expression using (62) we obtain (63) and our first claim is proved. Next we claim that $\left\langle E_{r} u^{*}, E_{r}^{*} u\right\rangle=2^{-d}\left\langle u^{*}, u\right\rangle$. To see this, observe by (1), (11), (12), Lemma 9.2(ii) and (63) that

$$
\begin{aligned}
\left\langle u^{*}, u\right\rangle & =\left\langle E_{r}^{*} u^{*}, u\right\rangle=\left\langle u^{*}, E_{r}^{*} u\right\rangle=\sum_{i=0}^{d}\left\langle E_{r+i} u^{*}, E_{r}^{*} u\right\rangle \\
& =\left\langle E_{r} u^{*}, E_{r}^{*} u\right\rangle \sum_{i=0}^{d}\left(\begin{array}{l}
d \\
i
\end{array}\right)=\left\langle E_{r} u^{*}, E_{r}^{*} u\right\rangle 2^{d}
\end{aligned}
$$

and the second claim follows. Combining the two claims we get the desired result.

(ii), (iii) Use (1), Lemma 7.3(i), Lemma 9.3 and (i) above.

Theorem 11.6 With reference to Notation 8.2, let $W$ denote an irreducible $T$-module with endpoint $r$ and diameter $d=D-2 r$. Then the following (i)-(iii) hold for $0 \leq$ $i, j \leq d$ and nonzero vectors $u \in E_{r} W, u^{*} \in E_{r}^{*} W, u^{\varepsilon} \in E_{r}^{\varepsilon} W$.

(i)

$$
\left\langle E_{r+i} u^{*}, E_{r+j}^{*} u^{\varepsilon}\right\rangle=\mathbf{i}^{j} 2^{-d}\left\langle u^{*}, u^{\varepsilon}\right\rangle\left(\begin{array}{l}
d \\
i
\end{array}\right)\left(\begin{array}{l}
d \\
j
\end{array}\right){ }_{2} F_{1}\left(\begin{array}{c}
-i,-j \\
-d
\end{array} ; 2\right) .
$$


(ii)

$$
\left\langle E_{r+i}^{*} u^{\varepsilon}, E_{r+j}^{\varepsilon} u\right\rangle=\mathbf{i}^{j} 2^{-d}\left\langle u^{\varepsilon}, u\right\rangle\left(\begin{array}{l}
d \\
i
\end{array}\right)\left(\begin{array}{l}
d \\
j
\end{array}\right){ }_{2} F_{1}\left(\begin{array}{c}
-i,-j \\
-d
\end{array} ; 2\right) .
$$

(iii)

$$
\left\langle E_{r+i}^{\varepsilon} u, E_{r+j} u^{*}\right\rangle=\mathbf{i}^{j} 2^{-d}\left\langle u, u^{*}\right\rangle\left(\begin{array}{l}
d \\
i
\end{array}\right)\left(\begin{array}{l}
d \\
j
\end{array}\right){ }_{2} F_{1}\left(\begin{array}{c}
-i,-j \\
-d
\end{array} ; 2\right) .
$$

PROOF. (i) By Theorem 11.2(ii) and (63),

$$
\left\langle E_{r+i} u^{*}, E_{r+j}^{*} u^{\varepsilon}\right\rangle=\mathbf{i}^{j}\left\langle E_{r} u^{*}, E_{r}^{*} u^{\varepsilon}\right\rangle\left(\begin{array}{l}
d \\
i
\end{array}\right)\left(\begin{array}{l}
d \\
j
\end{array}\right){ }_{2} F_{1}\left(\begin{array}{c}
-i,-j \\
-d
\end{array} ; 2\right) .
$$

We claim that $\left\langle E_{r} u^{*}, E_{r}^{*} u^{\varepsilon}\right\rangle=2^{-d}\left\langle u^{*}, u^{\varepsilon}\right\rangle$. To see this, observe by (1), (11), (12), Lemma 9.2(ii) and (67) that

$$
\begin{aligned}
\left\langle u^{*}, u^{\varepsilon}\right\rangle & =\left\langle E_{r}^{*} u^{*}, u^{\varepsilon}\right\rangle=\left\langle u^{*}, E_{r}^{*} u^{\varepsilon}\right\rangle=\sum_{i=0}^{d}\left\langle E_{r+i} u^{*}, E_{r}^{*} u^{\varepsilon}\right\rangle \\
& =\left\langle E_{r} u^{*}, E_{r}^{*} u^{\varepsilon}\right\rangle \sum_{i=0}^{d}\left(\begin{array}{l}
d \\
i
\end{array}\right)=\left\langle E_{r} u^{*}, E_{r}^{*} u^{\varepsilon}\right\rangle 2^{d}
\end{aligned}
$$

and the claim follows. Combining (67) with the claim we get the desired result.

(ii), (iii) Use (1), Lemma 7.3(i), Lemma 9.3 and (i) above.

Theorem 11.7 With reference to Notation 8.2, let $W$ denote an irreducible T-module with endpoint $r$ and diameter $d=D-2 r$. Then the following (i)-(iii) hold for $0 \leq$ $i, j \leq d$ and nonzero vectors $u \in E_{r} W, u^{*} \in E_{r}^{*} W, u^{\varepsilon} \in E_{r}^{\varepsilon} W$.

(i)

$$
\left\langle E_{r+i} u^{\varepsilon}, E_{r+j}^{*} u\right\rangle=\mathbf{i}^{i} 2^{-d}\left\langle u^{\varepsilon}, u\right\rangle\left(\begin{array}{l}
d \\
i
\end{array}\right)\left(\begin{array}{l}
d \\
j
\end{array}\right){ }_{2} F_{1}\left(\begin{array}{c}
-i,-j \\
-d
\end{array} ; 2\right) .
$$

(ii)

$$
\left\langle E_{r+i}^{*} u, E_{r+j}^{\varepsilon} u^{*}\right\rangle=\mathbf{i}^{i} 2^{-d}\left\langle u, u^{*}\right\rangle\left(\begin{array}{l}
d \\
i
\end{array}\right)\left(\begin{array}{l}
d \\
j
\end{array}\right){ }_{2} F_{1}\left(\begin{array}{c}
-i,-j \\
-d
\end{array} ; 2\right) .
$$

(iii)

$$
\left\langle E_{r+i}^{\varepsilon} u^{*}, E_{r+j} u^{\varepsilon}\right\rangle=\mathbf{i}^{i} 2^{-d}\left\langle u^{*}, u^{\varepsilon}\right\rangle\left(\begin{array}{l}
d \\
i
\end{array}\right)\left(\begin{array}{l}
d \\
j
\end{array}\right){ }_{2} F_{1}\left(\begin{array}{c}
-i,-j \\
-d
\end{array} ; 2\right) .
$$

Proof. (i) By Theorem 11.2(i),(ii) and (67),

$$
\left\langle E_{r+i} u^{\varepsilon}, E_{r+j}^{*} u\right\rangle=\mathbf{i}^{i}\left\langle E_{r} u^{\varepsilon}, E_{r}^{*} u\right\rangle\left(\begin{array}{l}
d \\
i
\end{array}\right)\left(\begin{array}{l}
d \\
j
\end{array}\right){ }_{2} F_{1}\left(\begin{array}{c}
-i,-j \\
-d
\end{array} ; 2\right) .
$$


We claim that $\left\langle E_{r} u^{\varepsilon}, E_{r}^{*} u\right\rangle=2^{-d}\left\langle u^{\varepsilon}, u\right\rangle$. To see this, observe by (1), (4), (5), Lemma 9.2(i) and (68) that

$$
\begin{aligned}
\left\langle u^{\varepsilon}, u\right\rangle & =\left\langle u^{\varepsilon}, E_{r} u\right\rangle=\left\langle E_{r} u^{\varepsilon}, u\right\rangle=\sum_{j=0}^{d}\left\langle E_{r} u^{\varepsilon}, E_{r+j}^{*} u\right\rangle \\
& =\left\langle E_{r} u^{\varepsilon}, E_{r}^{*} u\right\rangle \sum_{j=0}^{d}\left(\begin{array}{l}
d \\
j
\end{array}\right)=\left\langle E_{r} u^{\varepsilon}, E_{r}^{*} u\right\rangle 2^{d}
\end{aligned}
$$

and the claim follows. Combining (68) with the claim we get the desired result.

(ii), (iii) Use (1), Lemma 7.3(i), Lemma 9.3 and (i) above.

Theorem 11.8 With reference to Notation 8.2, let $W$ denote an irreducible $T$-module with endpoint $r$ and diameter $d=D-2 r$. Then the following (i)-(iii) hold for $0 \leq$ $i, j \leq d$ and nonzero vectors $u \in E_{r} W, u^{*} \in E_{r}^{*} W, u^{\varepsilon} \in E_{r}^{\varepsilon} W$.

(i)

$$
\left\langle E_{r+i} u^{*}, E_{r+j}^{\varepsilon} u^{*}\right\rangle=\mathbf{i}^{-i-j}(2-2 \mathbf{i})^{-d}\left\|u^{*}\right\|^{2}\left(\begin{array}{l}
d \\
i
\end{array}\right)\left(\begin{array}{l}
d \\
j
\end{array}\right){ }_{2} F_{1}\left(\begin{array}{c}
-i,-j \\
-d
\end{array} ; 2\right) .
$$

(ii)

$$
\left\langle E_{r+i}^{*} u^{\varepsilon}, E_{r+j} u^{\varepsilon}\right\rangle=\mathbf{i}^{-i-j}(2-2 \mathbf{i})^{-d}\left\|u^{\varepsilon}\right\|^{2}\left(\begin{array}{l}
d \\
i
\end{array}\right)\left(\begin{array}{l}
d \\
j
\end{array}\right){ }_{2} F_{1}\left(\begin{array}{c}
-i,-j \\
-d
\end{array} ; 2\right) .
$$

(iii)

$$
\left\langle E_{r+i}^{\varepsilon} u, E_{r+j}^{*} u\right\rangle=\mathbf{i}^{-i-j}(2-2 \mathbf{i})^{-d}\|u\|^{2}\left(\begin{array}{l}
d \\
i
\end{array}\right)\left(\begin{array}{l}
d \\
j
\end{array}\right){ }_{2} F_{1}\left(\begin{array}{c}
-i,-j \\
-d
\end{array} ; 2\right) .
$$

PROOF. (i) By Theorem 11.2(i),

$$
\left\langle E_{r+i} u^{*}, E_{r+j}^{\varepsilon} u^{*}\right\rangle=\frac{\left\|u^{*}\right\|^{2}}{\mathbf{i}^{i}(1-\mathbf{i})^{d}\left\langle u^{\varepsilon}, u^{*}\right\rangle}\left\langle E_{r+i} u^{\varepsilon}, E_{r+j}^{\varepsilon} u^{*}\right\rangle .
$$

The result now follows from Theorem 11.7(iii).

(ii), (iii) Use (1), Lemma 7.3(i), Lemma 9.3 and (i) above.

\section{The inner products between $u, u^{*}$ and $u^{\varepsilon}$}

With reference to Notation 8.2, let $W$ denote an irreducible $T$-module with endpoint $r$, and pick nonzero vectors $u \in E_{r} W, u^{*} \in E_{r}^{*} W, u^{\varepsilon} \in E_{r}^{\varepsilon} W$. In this section we display some equations involving the inner products between $u, u^{*}, u^{\varepsilon}$. 
Theorem 12.1 With reference to Notation 8.2, let $W$ denote an irreducible T-module with endpoint $r$ and diameter $d=D-2 r$. Then the following (i)-(iii) hold for nonzero vectors $u \in E_{r} W, u^{*} \in E_{r}^{*} W, u^{\varepsilon} \in E_{r}^{\varepsilon} W$.

(i) $\|u\|^{2}=(1+\mathbf{i})^{d}\left\langle u, u^{*}\right\rangle\left\langle u^{\varepsilon}, u\right\rangle\left\langle u^{\varepsilon}, u^{*}\right\rangle^{-1}$.

(ii) $\left\|u^{*}\right\|^{2}=(1+\mathbf{i})^{d}\left\langle u^{*}, u^{\varepsilon}\right\rangle\left\langle u, u^{*}\right\rangle\left\langle u, u^{\varepsilon}\right\rangle^{-1}$.

(iii) $\left\|u^{\varepsilon}\right\|^{2}=(1+\mathbf{i})^{d}\left\langle u^{\varepsilon}, u\right\rangle\left\langle u^{*}, u^{\varepsilon}\right\rangle\left\langle u^{*}, u\right\rangle^{-1}$.

Proof. (i) Using Theorem 11.2(iii) and Theorem 11.5(ii) we obtain

$$
\left\langle E_{r}^{*} u^{\varepsilon}, E_{r}^{\varepsilon} u\right\rangle=\frac{\|u\|^{2}\left\langle u^{\varepsilon}, u^{*}\right\rangle}{2^{d}(1+\mathbf{i})^{d}\left\langle u, u^{*}\right\rangle} .
$$

Comparing the above value for $\left\langle E_{r}^{*} u^{\varepsilon}, E_{r}^{\varepsilon} u\right\rangle$ with the value given in Theorem 11.6(ii) we obtain the desired result.

(ii), (iii) Similar to the proof of (i) above.

Corollary 12.2 With reference to Notation 8.2, let $W$ denote an irreducible $T$ module with endpoint $r$ and diameter $d=D-2 r$. Pick nonzero vectors $u \in E_{r} W$, $u^{*} \in E_{r}^{*} W, u^{\varepsilon} \in E_{r}^{\varepsilon} W$. Then the scalar

$$
\left\langle u, u^{*}\right\rangle\left\langle u^{*}, u^{\varepsilon}\right\rangle\left\langle u^{\varepsilon}, u\right\rangle(1+\mathbf{i})^{d}
$$

is real and positive.

Proof. By Theorem 12.1(i) the scalar (69) is equal to $\|u\|^{2}\left\langle u^{*}, u^{\varepsilon}\right\rangle\left\langle u^{\varepsilon}, u^{*}\right\rangle$. By construction $\|u\|^{2}$ is real and positive. Also $\left\langle u^{*}, u^{\varepsilon}\right\rangle\left\langle u^{\varepsilon}, u^{*}\right\rangle$ is real and positive since $\left\langle u^{\varepsilon}, u^{*}\right\rangle=\overline{\left\langle u^{*}, u^{\varepsilon}\right\rangle}$ by construction and $\left\langle u^{\varepsilon}, u^{*}\right\rangle \neq 0$ by Corollary 11.3. The result follows.

With reference to Theorem 12.1, the inner products $\left\langle u, u^{*}\right\rangle,\left\langle u^{*}, u^{\varepsilon}\right\rangle$ and $\left\langle u^{\varepsilon}, u\right\rangle$ are independent in the following sense.

Lemma 12.3 Let $a, b, c \in \mathbb{C}$ be such that abc $(1+\mathbf{i})^{d}$ is a positive real number. Then there exist nonzero vectors $u \in E_{r} W, u^{*} \in E_{r}^{*} W, u^{\varepsilon} \in E_{r}^{\varepsilon} W$ such that $\left\langle u, u^{*}\right\rangle=a$, $\left\langle u^{*}, u^{\varepsilon}\right\rangle=b,\left\langle u^{\varepsilon}, u\right\rangle=c$.

PROOF. Note that each of $a, b, c$ is nonzero. Pick arbitrary nonzero vectors $u_{1} \in$ $E_{r} W, u_{1}^{*} \in E_{r}^{*} W, u_{1}^{\varepsilon} \in E_{r}^{\varepsilon} W$ and define $\delta=a b c(1+\mathbf{i})^{d}\left\|c u_{1}^{*}\right\|^{-2}$. Also define $\lambda=$ $a \delta^{-1 / 2}\left\langle u_{1}, u_{1}^{*}\right\rangle^{-1}, \lambda^{*}=\delta^{1 / 2}, \lambda^{\varepsilon}=\bar{b} \delta^{-1 / 2}\left\langle u_{1}^{\varepsilon}, u_{1}^{*}\right\rangle^{-1}$. These scalars are well-defined by Corollary 11.3. We now define $u=\lambda u_{1}, u^{*}=\lambda^{*} u_{1}^{*}, u^{\varepsilon}=\lambda^{\varepsilon} u_{1}^{\varepsilon}$ and routinely obtain $\left\langle u, u^{*}\right\rangle=a,\left\langle u^{*}, u^{\varepsilon}\right\rangle=b,\left\langle u^{\varepsilon}, u\right\rangle=c$ using Theorem 12.1.

\section{The transition matrices}

In this section we display the transition matrices between the bases introduced in Section 9. We start with a comment. With reference to Notation 8.2, let $W$ denote an 
irreducible $T$-module with endpoint $r$ and diameter $d=D-2 r$. Let $u_{0}, \ldots, u_{d}$ and $v_{0}, \ldots, v_{d}$ denote bases for $W$. By the transition matrix from $u_{0}, \ldots, u_{d}$ to $v_{0}, \ldots, v_{d}$ we mean the matrix $C \in$ Mat $_{d+1}(\mathbb{C})$ which satisfies

$$
v_{j}=\sum_{i=0}^{d} C_{i j} u_{i} \quad(0 \leq j \leq d) .
$$

We recall a few properties of transition matrices. Let $C$ denote the transition matrix from $u_{0}, \ldots, u_{d}$ to $v_{0}, \ldots, v_{d}$. Then $C$ is invertible and $C^{-1}$ is the transition matrix from $v_{0}, \ldots, v_{d}$ to $u_{0}, \ldots, u_{d}$. If $u_{0}, \ldots, u_{d}$ are mutually orthogonal then the entries of $C$ are given by

$$
C_{i j}=\frac{\left\langle v_{j}, u_{i}\right\rangle}{\left\|u_{i}\right\|^{2}} \quad(0 \leq i, j \leq d) .
$$

In order to display the transition matrices in a compact form we abbreviate

$$
\Phi_{i j}=\left(\begin{array}{l}
d \\
j
\end{array}\right){ }_{2} F_{1}\left(\begin{array}{c}
-i,-j \\
-d
\end{array} ; 2\right)
$$

and

$$
D_{1}=\operatorname{diag}\left(\mathbf{i}^{0}, \mathbf{i}^{1}, \ldots, \mathbf{i}^{d}\right), \quad D_{2}=\operatorname{diag}\left(\mathbf{i}^{-0}, \mathbf{i}^{-1}, \ldots, \mathbf{i}^{-d}\right) .
$$

Theorem 13.1 With reference to Notation 8.2, let $W$ denote an irreducible $T$-module with endpoint $r$ and diameter $d=D-2 r$. Pick nonzero vectors $u \in E_{r} W, u^{*} \in E_{r}^{*} W$, $u^{\varepsilon} \in E_{r}^{\varepsilon} W$. Then the transition matrices between the bases (45) - (50) are given in Tables 1 and 2.

ProOf. Combine Theorem 11.1, Theorems 11.4 - 11.8 and (70).

Table 1

to the basis (45)

$\operatorname{diag}(1,1, \ldots, 1)$

basis (45)

from the

basis (46)

from the

basis (47)

from the

basis (48)

from the

basis (49)

from the

basis (50) to the basis (46)

to the basis (47)

$\frac{1}{(1-\mathbf{i})^{d}}\left[\begin{array}{ll}\mathbf{i}^{-i-j} & \Phi_{i j}\end{array}\right]_{i, j=0}^{d}$

$\frac{\left\langle u^{*}, u\right\rangle}{\|u\|^{2}}\left[\mathbf{i}^{-i} \Phi_{i j}\right]_{i, j=0}^{d}$

$\operatorname{diag}(1,1, \ldots, 1)$

$\frac{(1-\mathbf{i})^{d}\left\langle u^{*}, u\right\rangle}{\|u\|^{2}} D_{1}$

$\frac{1}{(1+\mathbf{i})^{d}}\left[\mathbf{i}^{i+j} \Phi_{i j}\right]_{i, j=0}^{d}$

$\frac{(1+\mathbf{i})^{d}\left\langle u, u^{*}\right\rangle}{\left\|u^{*}\right\|^{2}} D_{2}$

$\operatorname{diag}(1,1, \ldots, 1)$

$$
\frac{\left\langle u, u^{*}\right\rangle}{\left\|u^{*}\right\|^{2}}\left[\mathbf{i}^{j} \Phi_{i j}\right]_{i, j=0}^{d}
$$

$\frac{\left\langle u, u^{*}\right\rangle}{\left\|u^{*}\right\|^{2}}\left[\mathbf{i}^{i} \Phi_{i j}\right]_{i, j=0}^{d}$

$\frac{1}{(1+\mathbf{i})^{d}}\left[\mathbf{i}^{i+j} \Phi_{i j}\right]_{i, j=0}^{d}$

$\frac{\left\langle u, u^{*}\right\rangle}{\left\|u^{*}\right\|^{2}}\left[\Phi_{i j}\right]_{i, j=0}^{d}$

$\frac{\left\langle u, u^{\varepsilon}\right\rangle}{\left\|u^{\varepsilon}\right\|^{2}}\left[\Phi_{i j}\right]_{i, j=0}^{d}$

$\frac{\left\langle u^{*}, u^{\varepsilon}\right\rangle}{\left\|u^{\varepsilon}\right\|^{2}}\left[\mathbf{i}^{j} \Phi_{i j}\right]_{i, j=0}^{d}$

$\frac{\left\langle u, u^{\varepsilon}\right\rangle}{\left\|u^{\varepsilon}\right\|^{2}}\left[\mathbf{i}^{-i} \Phi_{i j}\right]_{i, j=0}^{d}$

$\frac{\left\langle u, u^{\varepsilon}\right\rangle}{\left\|u^{\varepsilon}\right\|^{2}}\left[\mathbf{i}^{-j} \Phi_{i j}\right]_{i, j=0}^{d}$

$\frac{\left\langle u^{*}, u^{\varepsilon}\right\rangle}{\left\|u^{\varepsilon}\right\|^{2}}\left[\Phi_{i j}\right]_{i, j=0}^{d}$ 
Table 2
to the basis (48)
to the basis (49)
to the basis (50)

\begin{tabular}{|c|c|c|c|}
\hline $\begin{array}{l}\text { from the } \\
\text { basis (45) }\end{array}$ & $\frac{\left\langle u^{*}, u\right\rangle}{\|u\|^{2}}\left[\Phi_{i j}\right]_{i, j=0}^{d}$ & $\frac{\left\langle u^{\varepsilon}, u\right\rangle}{\|u\|^{2}}\left[\mathbf{i}^{j} \Phi_{i j}\right]_{i, j=0}^{d}$ & $\frac{(1+\mathbf{i})^{d}\left\langle u^{\varepsilon}, u\right\rangle}{\|u\|^{2}} D_{2}$ \\
\hline $\begin{array}{l}\text { from the } \\
\text { basis (46) }\end{array}$ & $\frac{\left\langle u^{*}, u\right\rangle}{\|u\|^{2}}\left[\mathbf{i}^{-j} \Phi_{i j}\right]_{i, j=0}^{d}$ & $\frac{\left\langle u^{\varepsilon}, u\right\rangle}{\|u\|^{2}}\left[\Phi_{i j}\right]_{i, j=0}^{d}$ & $\frac{\left\langle u^{\varepsilon}, u\right\rangle}{\|u\|^{2}}\left[\mathbf{i}^{i} \Phi_{i j}\right]_{i, j=0}^{d}$ \\
\hline $\begin{array}{l}\text { from the } \\
\text { basis (47) }\end{array}$ & $\frac{1}{(1-\mathbf{i})^{d}}\left[\mathbf{i}^{-i-j} \Phi_{i j}\right]_{i, j=0}^{d}$ & $\frac{\left\langle u^{\varepsilon}, u^{*}\right\rangle}{\left\|u^{*}\right\|^{2}}\left[\mathbf{i}^{-i} \Phi_{i j}\right]_{i, j=0}^{d}$ & $\frac{\left\langle u^{\varepsilon}, u^{*}\right\rangle}{\left\|u^{*}\right\|^{2}}\left[\Phi_{i j}\right]_{i, j=0}^{d}$ \\
\hline $\begin{array}{l}\text { from the } \\
\text { basis (48) }\end{array}$ & $\operatorname{diag}(1,1, \ldots, 1)$ & $\frac{(1-\mathbf{i})^{d}\left\langle u^{\varepsilon}, u^{*}\right\rangle}{\left\|u^{*}\right\|^{2}} D_{1}$ & $\frac{\left\langle u^{\varepsilon}, u^{*}\right\rangle}{\left\|u^{*}\right\|^{2}}\left[\mathbf{i}^{-j} \Phi_{i j}\right]_{i, j=0}^{d}$ \\
\hline $\begin{array}{l}\text { from the } \\
\text { basis (49) }\end{array}$ & $\frac{(1+\mathbf{i})^{d}\left\langle u^{*}, u^{\varepsilon}\right\rangle}{\left\|u^{\varepsilon}\right\|^{2}} D_{2}$ & $\operatorname{diag}(1,1, \ldots, 1)$ & $\frac{1}{(1-\mathbf{i})^{d}}\left[\mathbf{i}^{-i-j} \Phi_{i j}\right]_{i, j=0}^{d}$ \\
\hline $\begin{array}{l}\text { from the } \\
\text { basis }(50)\end{array}$ & $\frac{\left\langle u^{*}, u^{\varepsilon}\right\rangle}{\left\|u^{\varepsilon}\right\|^{2}}\left[\begin{array}{ll}\mathbf{i}^{i} & \Phi_{i j}\end{array}\right]_{i, j=0}^{d}$ & $\frac{1}{(1+\mathbf{i})^{d}}\left[\mathbf{i}^{i+j} \Phi_{i j}\right]_{i, j=0}^{d}$ & $\operatorname{diag}(1,1, \ldots, 1)$ \\
\hline
\end{tabular}

Acknowledgement The author would like to thank Paul Terwilliger for proposing the problem and for his careful reading of the earlier versions of this manuscript.

\section{References}

1. Andrews, G.E., Askey, R., Roy, R.: Special Functions. Encyclopedia of Mathematics and Its Applications. Cambridge University Press, Cambridge (1999)

2. Bannai, E., Ito, T.: Algebraic Combinatorics I: Association Schemes. Benjamin-Cummings Lecture Notes, vol. 58. Benjamin-Cummings, Menlo Park (1984)

3. Brouwer, A.E., Cohen, A.M., Neumaier, A.: Distance-Regular Graphs. Springer, Berlin, Heidelberg (1989)

4. Caughman IV, J.S.: The Terwilliger algebras of bipartite $P$ - and $Q$-polynomial schemes. Discrete Math. 196, 65-95 (1999)

5. Curtin, B.: Modular Leonard triples. Linear Algebra Appl. 424, 510-539 (2007)

6. Curtin, B.: Spin Leonard pairs. Ramanujan J. 13, 319-332 (2006)

7. Curtin, B.: Distance-regular graphs that support a spin model are thin. Discrete Math. 197-198, 205216 (1999)

8. Curtin, B., Nomura, K.: Distance-regular graphs related to the quantum enveloping algebra of $s l(2)$. J. Algebr. Comb. 12, 25-36 (2000)

9. Eves, H.: Elementary Matrix Theory. Allyn and Bacon, Boston (1966)

10. Go, J.T.: The Terwilliger algebra of the hypercube. Eur. J. Comb. 23, 399-429 (2002)

11. Godsil, C.D.: Algebraic Combinatorics. Chapman and Hall, New York (1993)

12. Ito, T., Tanabe, K., Terwilliger, P.: Some algebra related to $P$ - and $Q$-polynomial association schemes. DIMACS Ser. Discrete Math. Theor. Comput. Sci. 56, 167-192 (2001)

13. Koekoek, R., Swarttouw, R.F.: The Askey-scheme of hypergeometric orthogonal polynomials and its q-analogue. Delft University of Technology, Faculty of Information Technology and Systems, Department of Technical Mathematics and Informatics, Report no. 98-17 (1998). http://fa.its.tudelft.nl/ koekoek/askey/

14. Koelink, H.T.: Askey-Wilson polynomials and the quantum $s u(2)$ group: survey and applications. Acta. Appl. Math. 44, 295-352 (1996)

15. Korovnichenko, A., Zhedanov, A.: Classical Leonard triples. Preprint

16. Schrijver, A.: New code upper bounds from the Terwilliger algebra and semidefinite programming. IEEE Trans. Inf. Theory 51, 2859-2866 (2005)

17. Terwilliger, P.: The subconstituent algebra of an association scheme, part I. J. Algebr. Comb. 1, 363388 (1992) 
18. Terwilliger, P.: The subconstituent algebra of an association scheme, part II. J. Algebr. Comb. 2, 73103 (1993)

19. Terwilliger, P.: The subconstituent algebra of an association scheme, part III. J. Algebr. Comb. 2, 177-210 (1993)

20. Terwilliger, P.: Two relations that generalize the $q$-Serre relations and the Dolan-Grady relations. In: Physics and Combinatorics, Nagoya, 1999, pp. 377-398. World Scientific, River Edge (2001)

21. Terwilliger, P.: Two linear transformations each tridiagonal with respect to an eigenbasis of the other. Linear Algebra Appl. 330, 149-203 (2001)

22. Terwilliger, P.: Leonard pairs from 24 points of view. Rocky Mt. J. Math. 32(2), 827-888 (2002)

23. Terwilliger, P.: Introduction to Leonard pairs. J. Comput. Appl. Math. 153(2), 463-475 (2003)

24. Terwilliger, P.: Leonard pairs and the $q$-Racah polynomials. Linear Algebra Appl. 387, 235-276 (2004)

25. Terwilliger, P.: Two linear transformations each tridiagonal with respect to an eigenbasis of the other; Comments on the parameter array. Des. Codes Cryptogr. 34, 307-332 (2005)

26. Terwilliger, P.: Two linear transformations each tridiagonal with respect to an eigenbasis of the other: comments on the split decomposition. J. Comput. Appl. Math. 178, 437-452 (2005)

27. Terwilliger, P.: Two linear transformations each tridiagonal with respect to an eigenbasis of the other; the TD-D canonical form and the LB-UB canonical form. J. Algebra 291, 1-45 (2005)

28. Zhedanov, A.S.: "Hidden symmetry" of Askey-Wilson polynomials. Teor. Mat. Fiz. 89, 190-204 (1991) 